\title{
Estimating the incidence of breast cancer in Africa: a systematic review and meta-analysis
}

\author{
Davies Adeloye ${ }^{1,2,3}$, Olaperi Y. Sowunmi ${ }^{4}$, \\ Wura Jacobs ${ }^{5}$, Rotimi A David ${ }^{6}$, Adeyemi \\ A Adeosun7, Ann O. Amuta ${ }^{8}$, Sanjay \\ Misra $^{9}$, Muktar Gadanya ${ }^{10}$, Asa Auta ${ }^{11}$, \\ Michael O Harhay ${ }^{12,13}$, Kit Yee Chan ${ }^{3}$ \\ ${ }^{1}$ Nigerian Urban Reproductive Health Initiative, Abuja, \\ Nigeria \\ 2 Johns Hopkins Centre for Communication Programs, \\ Baltimore, Maryland, USA \\ ${ }^{3}$ Centre for Global Health Research and the World \\ Health Organization Collaborating Centre for \\ Population Health Research and Training, Usher \\ Institute, University of Edinburgh, Scontland, UK \\ ${ }^{4}$ Computer and Information Sciences, Covenant \\ University, Ota, Ogun State, Nigeria \\ ${ }^{5}$ Department of Health Science, California State \\ University, Fullerton, California, USA \\ ${ }^{6}$ Department of Urology, Morriston Hospital, Abertawe \\ Bro Morgannwg University Health Board, Swansea, UK \\ ${ }^{7}$ Health Initiative Department, National Jewish Hospital, \\ Denver, Colorado, USA \\ ${ }^{8}$ Department of Health Studies, Texas Woman's \\ University, Denton, Texas, USA \\ ${ }^{9}$ Department of Computer Engineering, Atilim \\ University, Turkey \\ ${ }^{10}$ Department of Community Medicine, Aminu Kano \\ Teaching Hospital, Bayero University, Kano, Nigeria \\ ${ }^{11}$ School of Pharmacy and Biomedical Sciences, \\ University of Central Lancashire, Fylde Road, Preston, \\ UK \\ ${ }^{12}$ Department of Biostatistics, Epidemiology and \\ Informatics, Perelman School of Medicine, University \\ of Pennsylvania, Philadelphia, Pennsylvania, USA \\ ${ }^{13}$ Palliative and Advanced Illness Research (PAIR) \\ Center, Perelman School of Medicine, University of \\ Pennsylvania, Philadelphia, Pennsylvania, USA
}

\section{Correspondence to:}

Dr. Davies Adeloye

Nigerian Urban Reproductive Health Initiative

No 10 Aderemi Adesoji Street

Abuja

Nigeria

bummyadeloy@gmail.com
Background Breast cancer is estimated to be the most common cancer worldwide. We sought to assemble publicly available data from Africa to provide estimates of the incidence of breast cancer on the continent.

Methods A systematic search of Medline, EMBASE, Global Health and African Journals Online (AJOL) was conducted. We included population- or hospital-based registry studies on breast cancer conducted in Africa, and providing estimates of the crude incidence of breast cancer among women. A random effects meta-analysis was employed to determine the pooled incidence of breast cancer across studies.

Results The literature search returned 4648 records, with 41 studies conducted across 54 study sites in 22 African countries selected. We observed important variations in reported cancer incidence between population- and hospital-based cancer registries. The overall pooled crude incidence of breast cancer from population-based registries was 24.5 per 100000 person years (95\% confidence interval (CI) 20.1-28.9). The incidence in North Africa was higher at 29.3 per 100000 (95\% CI 20.0-38.7) than Sub-Saharan Africa (SSA) at 22.4 per 100000 (95\% CI 17.2-28.0). In hospital-based registries, the overall pooled crude incidence rate was estimated at 23.6 per 100000 (95\% CI 18.5-28.7). SSA and Northern Africa had relatively comparable rates at 24.0 per 100000 (95\% CI 17.5-30.4) and 23.2 per 100000 (95\% CI 6.6-39.7), respectively. Across both registries, incidence rates increased considerably between 2000 and 2015.

Conclusions The available evidence suggests a growing incidence of breast cancer in Africa. The representativeness of these estimates is uncertain due to the paucity of data in several countries and calendar years, as well as inconsistency in data collation and quality across existing cancer registries.

Cancer is a leading cause of morbidity and mortality among women globally, accounting for about 17.5 million cancer cases and 9 million deaths in 2015 [1]. Breast cancer is now ranked the most common cancer worldwide, increasing from 1.7 million incident cases in 2005 to 2.4 million cases in 2015 [2]. In- 
sights into the epidemiology and risks associated with breast cancer have seen relative improvements in the response to breast cancer across population groups, particularly in high-income settings [3]. However, in Africa and many low- and middle-income countries (LMICs), several challenges including poor health infrastructure, incomplete vital registrations, lack of population awareness, delayed health seeking behavior and low levels of female education and empowerment have led to high mortality from breast cancer [4], and also engendered a complex barrier to improving public health response to breast cancer in these settings [5].

The International Agency for Cancer Research (IARC) estimated that the incidence of breast cancer ranged from 27 per 100000 women in central Africa to 39 per 100000 women in southern Africa in 2012 [6]. Although, the incidence of breast cancer appears to be relatively low in Sub-Saharan Africa (SSA), survival from the disease is also generally poor in the region, with high mortality recorded in many settings $[6,7]$. The poor survival of breast cancer patients in SSA has been associated with late presentation, poor health care infrastructure and lack of adequate funding, amidst other competing public health challenges [5]. Moreover, in isolated SSA countries with relatively good breast cancer care services, inequity and unaffordability of the services provided have been widely reported $[5,8]$. Consequently, late presentations and advanced stages at diagnosis of several breast cancer cases have been prevalent, likely explaining the higher mortality rates reported [7]. With ageing, population growth, and adoption of unhealthy lifestyles, the burden of breast cancer is projected to double in Africa by 2030 [9], especially in the absence of effective public health policies and interventions [9]. See Box 1 for an outline of what is already known, and what this study adds.

Breast cancer prevention and control in Africa is relatively limited, owing in part to a lack of reliable epidemiologic risk factor data and information from which evidence-based interventions could have been developed $[10,11]$. For example, out of 46 World Health Organization (WHO) member states in SSA, only 20 (43.4\%) have active cancer registries [12], spanning a wide range of coverage and completeness. These registries are poorly funded, largely limited to specific sub-national population groups (or even not population-based), and often do not meet standards required for active collation of cancer data [12]. Moreover, IARC also reported that due to lack of data on cancer incidence and mortality across developing countries, cancer estimates in these countries have been derived from frequency data or rates from neighboring countries with contextual similarities [9]. These efforts have no doubt contributed to the understanding of cancer burden in these settings [13]. However, intensifying ongoing efforts in the active collation of data from population- and hospital-based cancer registries across Africa and many developing countries may encourage further cancer-related research activities toward improving the overall representativeness of estimates provided $[14,15]$. Based on publicly available data, we estimated the incidence of breast cancer in Africa separately from population- and hospital-based cancer registries. We also examined the potential implication of the quality of data available from these registries on future responses to breast cancer in the region.

Box 1. About the study

\section{What is already known?}

The International Association of Cancer Registries (IACR) has been the main sources of cancer data in Africa, particularly by aiding African countries towards establishment of cancer registries. North Africa appears to have more robust data on cancer, with estimated incidence rates of breast cancer across North African states comparable to some high-income settings. In SSA, current reports reveal an increasing number of breast cancer cases diagnosed in the ages 35-49 years, with many presenting at advanced late-stage disease. Largely, data availability remains an ongoing limitation in understanding and estimating the incidence of breast cancer on the African continent.

\section{What this study adds}

This study provides the first systematic review and meta-analysis of publicly available evidence on the incidence of female breast cancer in Africa. There is limited data on cancer in Africa, particularly in Central Africa; only 22 African countries were included in this study. Population-based cancer registries remain the main sources of data on breast cancer in Africa as these provided 67\% of all data points. Our estimated incidence of breast cancer increased between 2000 and 2015 across both registries, suggestive of a rising breast cancer incidence in Africa. The mean age of populations covered ranged from 30.6 to 60.8 years, with over $33 \%$ and $81 \%$ of population in ages 30-49 years, and 30-59 years, respectively. 


\section{METHODS}

\section{Search strategy and data sources}

We conducted an initial scoping literature search to identify Medical Subject Headings (MESH) and relevant keywords, following which a final search strategy was developed. We conducted a systematic search of Medline, EMBASE, Global Health and African Journals Online (AJOL), with search dates set from January 1980 to December 2016. We also conducted additional searches of Google Scholar, and websites of the International Association of Cancer Registries (IACR) and WHO African Region (AFRO). We equally reviewed the "GLOBOCAN studies" [6,9,16], "Cancer Incidence in Five Continents (CI5) series" [17], and "Cancer in Africa: Epidemiology and Prevention" [18] for more studies or additional data for studies already selected. Reference lists of initially selected studies were further hand-searched. The list of African countries was based on the World Bank list of economies [19] (Table 1).

\section{Selection criteria}

We included population-based or hospital-based cancer registry studies on breast cancer conducted primarily on African population groups, and providing crude estimates of the cases or incidence of breast cancer among women in the population over a specified period. Studies were included if they were completed on or after the year 2000, to give a fair representation of the current burden. Hospital-based studies that only reported cases of breast cancer were also included if they provided sufficient information on the reference population to allow an estimation of the population or person-years at risk. We excluded studies on non-human subjects, and those that were mainly reviews, case reports, opinions or editorials. No language restrictions were applied.

\section{Confirmation of breast cancer diagnosis}

We included studies that identified breast cancer based on histological diagnosis with or without i) clinical evaluation by a physician, ii) radiological investigations (mammography, breast ultrasound, computed tomography scan or magnetic resonance imaging), iii) laboratory tests (estrogen or progesterone receptor (ER or PR) status, the human epidermal growth factor receptor 2 (HER2) status, and breast cancer antigen (BRCA1 or BRCA2) or iv) community reported cases.

\section{Quality criteria}

Each study was assessed for quality according to a set of five (5) predefined criteria. The first criterion was based on the cancer registration process. This considered how cancer registries collated data and the approach employed for data ascertainment. The second considered the coding criteria employed across studies to determine if the reported cancer types were classified according to the primary anatomic site

Table 1. Search terms for studies on breast cancer in Africa

\begin{tabular}{cl} 
NuMBER & SEARGHES \\
& $\begin{array}{l}\text { africa/ or africa, northern/ or algeria/ or egypt/ or libya/ or morocco/ or africa, central/ or cameroon/ or central african } \\
\text { republic/ or chad/ or congo/ or "democratic republic of the congo"/ or equatorial guinea/ or gabon/ or africa, eastern/ or } \\
\text { burundi/ or djibouti/ or eritrea/ or ethiopia/ or kenya/ or rwanda/ or somalia/ or sudan/ or tanzania/ or uganda/ or africa, } \\
\text { southern/ or angola/ or botswana/ or lesotho/ or malawi/ or mozambique/ or namibia/ or south africa/ or swaziland/ or } \\
\text { zambia/ or zimbabwe/ or africa, western/ or benin/ or burkina faso/ or cape verde/ or cote d'ivoire/ or gambia/ or ghana/ } \\
\text { or guinea/ or guinea-bissau/ or liberia/ or mali/ or mauritania/ or niger/ or nigeria/ or senegal/ or sierra leone/ or togo/ }\end{array}$ \\
\hline 2 & exp vital statistics/ or exp incidence/ \\
\hline 3 & (incidence* or prevalence* or morbidity or mortality).tw. \\
\hline 4 & (disease adj3 burden).tw. \\
\hline 5 & exp "cost of illness"/ \\
\hline 6 & exp quality-adjusted life years/ \\
\hline 7 & QALY.tw. \\
\hline 8 & Disability adjusted life years.mp. \\
\hline 9 & (initial adj2 burden).tw. \\
\hline 10 & exp risk factors/ \\
\hline 11 & 2 or 3 or 4 or 5 or 6 or 7 or 8 or 9 or 10 \\
\hline 12 & exp breast cancer/ \\
\hline 12 & 1 and 11 and 12 \\
\hline
\end{tabular}


(topography) or cellular characteristics (morphology—histology, behaviour, and grade) using the international classification of diseases (ICD) and oncology (ICD-O) guidelines [20-22]. The third assessed how population or person-years at risk were generated in each study. The fourth and fifth criteria checked whether the population covered in each study was representative of the target (subnational) and national populations, respectively. Each criterion was scored one (1), and studies were finally graded as high (45), moderate (2-3) or low (0-1) quality based on the number of criteria they met (Table S1 in Online Supplementary Document). All low-quality studies were excluded from the review.

\section{Data extraction}

Two reviewers (OYS and AA) independently screened studies against the inclusion and exclusion criteria and performed the data extraction. Any disagreement over article inclusion, exclusion or data extraction between the two initial reviewers was resolved through a final assessment by a third reviewer (DA). As we already employed a two-stage search and extraction process based on a combination of independent review and reassessment, we did not calculate Kappa statistics to determine agreement between the reviewers. We extracted data and relevant information systematically from each study. This included location, period, design, cancer registry, confirmation of diagnosis, data collation methods, coding criteria, data ascertainment and modality with which population or person years at risk were generated. The basic numerical data extracted were mean age (or age range), population or person years at risk, cancer cases, and crude incidence. For studies conducted on the same cancer registry over the same period, we selected the first chronologically published study, and supplementary data from other studies were added to the selected paper. All extracted data were separated into hospital-based and population-based studies, further sorted based on different regions in Africa (central, east, north, south, and west), and stored in Excel 2013 (Microsoft Inc, Redmond, WA, USA).

\section{Data analysis}

From extracted crude incidence rates of breast cancer, we conducted a random effects meta-analysis (DerSimonian and Laird method) [23]. For studies not reporting population or person years at risk, we checked the UN population projections of the country for the study period and multiplied by the years covered. For sub-national populations, we applied the subnational population ratio to standardize the United Nations (UN) projections of the country for the study period. Standard errors were estimated from the crude incidence rates and person years assuming a Poisson distribution. Crude meta-estimates and confidence intervals (expressed per 100000 person-years) were pooled from individual crude incidence rates and reported by registry-type for the African continent, the African sub-regions, age groups, and the years 2000 and 2015. I-squared ( $\left.\mathrm{I}^{2}\right)$ statistics and subgroup (sensitivity) analysis were conducted to assess heterogeneity between studies. We conducted a meta-regression analysis (with age and year as confounders) to further check consistency of the data extracted and estimates reported for hospital-based and population-based registries, respectively. All statistical analyses were conducted on Stata 13.1 (Stata Corp LP, College Station, TX, USA).

\section{RESULTS}

\section{Systematic review}

The literature search returned a total of 4648 studies. There were 4633 studies identified from the databases-Medline (1035), EMBASE (2811), Global Health (733) and AJOL (54). 15 additional studies were identified from Google Scholar, IARC and WHO AFRO websites, and hand-searching of reference lists. A total of 2858 records remained after duplicates were excluded. After reviewing titles for relevance (ie, studies providing incidence of breast cancer in any African location), 2650 studies were excluded, giving a total of 208 full texts that were assessed. After applying the selection criteria, 167 studies were excluded. A total of 41 studies were finally retained for the review [24-64] (Figure 1).

\section{Study characteristics}

The 41 studies retained were conducted across 54 study sites (locations) in 22 African countries (Table 2 ). West Africa had the highest number of study sites (16) with seven of these sites located in Nigeria. South Africa closely followed with 6 sites while others were Uganda and Tunisia ( 5 sites each), Morocco (4 sites), Algeria, Ghana, and Libya (3 sites each), and Cameroon, Egypt, Mozambique and Niger (2 sites 


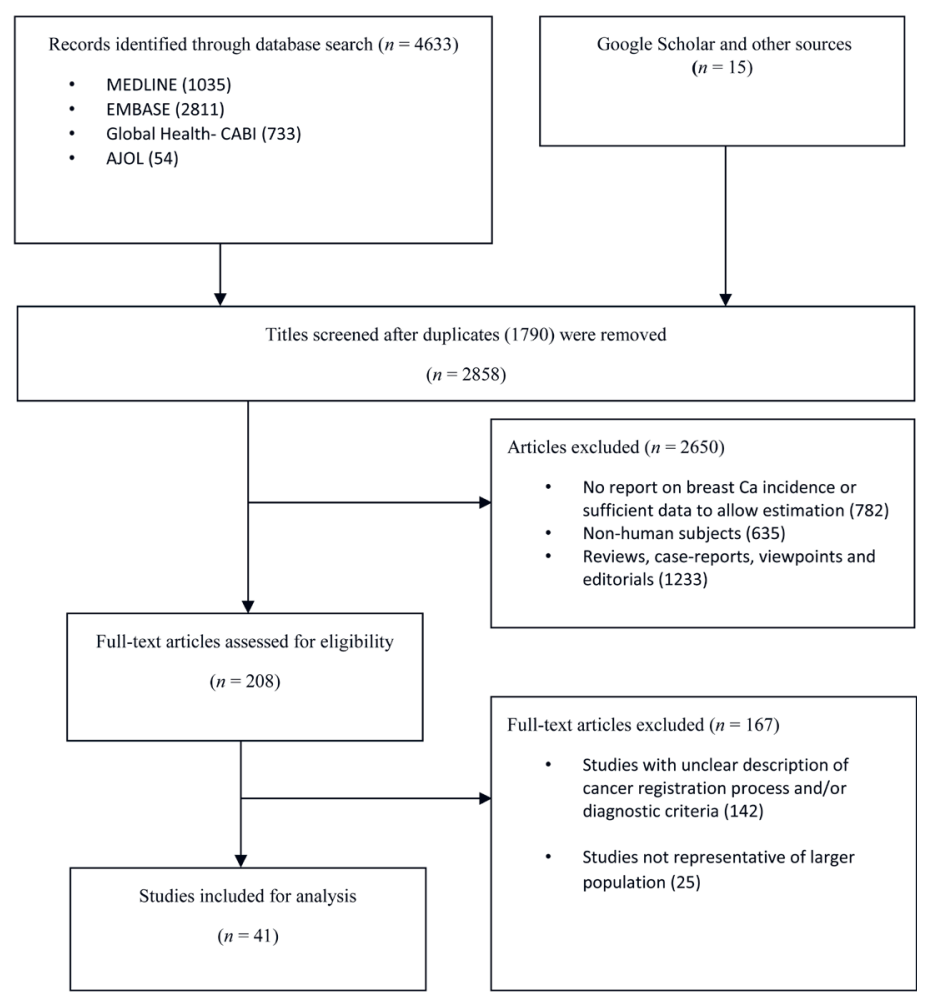

Figure 1. PRISMA flowchart of search strategy.

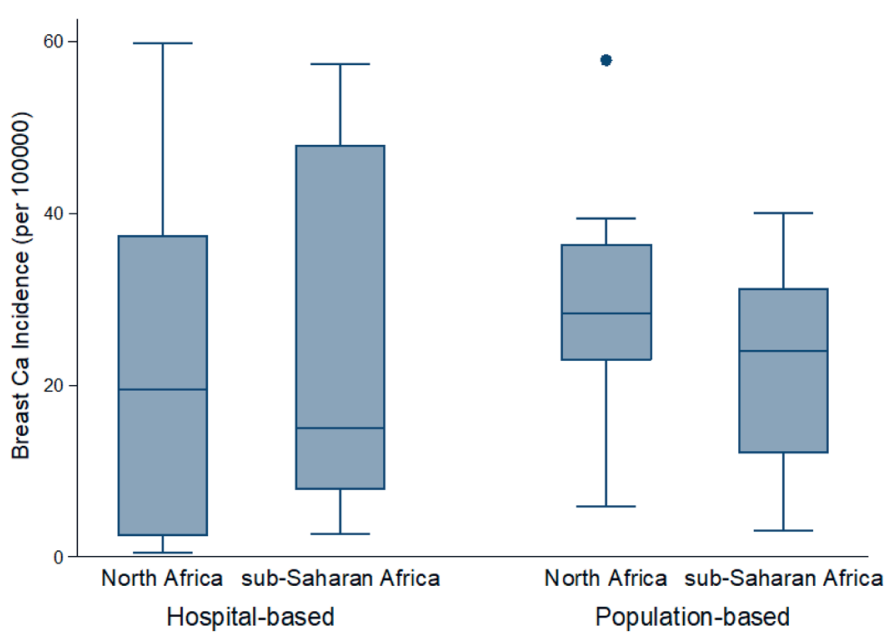

Figure 2. Distribution of crude incidence rates by registry type and African sub-region. each). In total, SSA had 36 sites (70\%) while North Africa had 18 sites (30\%) (see Table 2 for details). Population-based registries accounted for $67 \%$ of all study sites. These population-based registries reported mainly active or passive case findings across referral hospitals, pathology laboratories, and communities within the geographical remit of the cancer registry. Data were abstracted mostly from pre-designed pathology/laboratory forms confirming breast cancer diagnosis, with all sites reporting morphological/histological verification of the breast cancer cases. Mean age of study population ranged from 30.6 to 60.8 years (median 50.2 years), with over 33\% in the age group 30-49 years, and over $81 \%$ in $30-59$ years (Table 2 ).

Following the quality assessment, 23 (56\%) studies were graded high, of which (20) $87 \%$ were conducted in population-based registries, which suggests the internal consistency and validity of these registries. However, most hospital-based studies were of moderate quality, as 15 (83\%) of the total 18 studies graded moderate were conducted in hospital-based registries (Figure 2, Table 2, Table S1 in Online Supplementary Document). The basic limitations of many hospital-based registries were related to poorly defined data collation and ascertainment process. Moreover, catchment areas were also not well-defined, with this affecting the estimation of person-years across these registries.

\section{Pooled crude incidence rate of breast cancer in Africa}

\section{Population-based registries}

From population-based registries (36 study sites), the overall pooled crude incidence of breast cancer in Africa was 24.5 per 100000 person years (95\% Confidence Interval [CI]: 20.1-28.9) (Figure 3, plate A). The incidence rate in North Africa was higher at 29.3 per 100000 (95\% CI 20.0-38.7) than the pooled rates in SSA at 22.4 per 100000 (95\% CI 17.2-28.0). The estimated incidence rates in East Africa and West Africa were also relatively higher at 28.0 (95\% CI 21.7-33.7) and 24.2 (95\% CI 15.4-33.0) per 100000 , respectively. Southern Africa had a pooled rate of 19.0 (95\% CI 10.1-27.8), while Central Africa had the lowest pooled incidence rates at 13.4 (95\% CI 7.2-34.1) per 100000.

Due to high heterogeneity across studies (99.9\%), we also reported the median incidence of breast cancer from all population-based registries, estimated at 28.1 per 100000 , with an interquartile range (IQR) of 18.6-31.3 per 100000 (Table 3, Figure 2). Besides, a sensitivity analysis based on quality criteria showed studies graded as high quality had almost similar pooled incidence as the overall pooled incidence at 24.3 per 100000 person years (95\% CI 19.9-28.8), compared to moderate quality studies at 26.1 per 100000 (95\% CI 5.5-46.7) (Figure 3, plate B).

Moreover, in the population-based registries, we observed an increasing incidence with increasing age of subjects and over the study period covered (Table 3 ). The pooled incidence rate was highest among persons older than 60 years at 36.6 per 100000 (95\% CI 30.7-42.4) compared to an incidence rate of 3.3 per 100000 (95\% CI 2.7-3.8) among persons aged 30-39 years (Table 3). Between 2000 and 2015, the 
A.

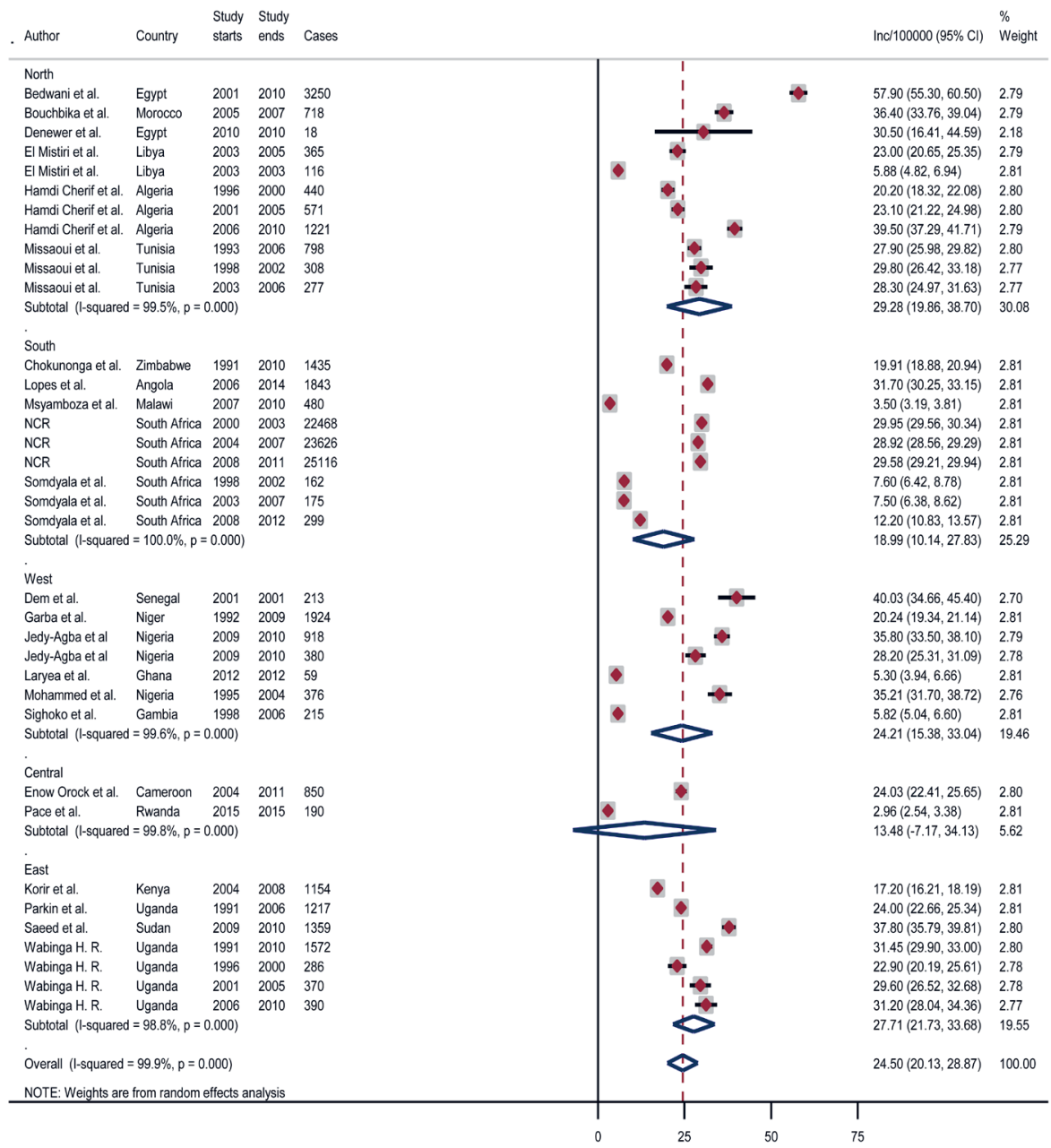

B.

\begin{tabular}{|c|c|c|c|c|c|}
\hline Author & County & $\begin{array}{l}\text { Study Study } \\
\text { starts } \\
\text { ends Cases }\end{array}$ & & & Inc/100000 (95\% CII) Weignt \\
\hline $\begin{array}{l}\text { High } \\
\text { Bectaraie tal }\end{array}$ & & & & & \\
\hline $\begin{array}{l}\text { Bouchbok e e tal. } \\
\text { Choluknga el t. }\end{array}$ & $\begin{array}{l}\text { Morococo } \\
\text { Zurimbawe }\end{array}$ & $\begin{array}{lll}2005 & 2007 & 718 \\
1991 & 2010 & 1435\end{array}$ & $*$ & & 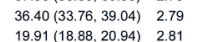 \\
\hline 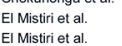 & $\begin{array}{l}\text { Lithoua } \\
\text { Libya } \\
\text { Lyba }\end{array}$ & 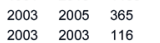 & 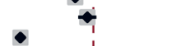 & & $\begin{array}{lll}2.300(20.065,25.35) & 2.79 \\
5.88(4.82,69.94) & 2.81\end{array}$ \\
\hline $\begin{array}{l}\text { Enow orock et al. } \\
\text { Garas a tal }\end{array}$ & $\begin{array}{l}\text { Cameroon } \\
\text { Niger }\end{array}$ & $\begin{array}{lll}2004 & 2011 & 850 \\
1992 & 2009 & 1924\end{array}$ & $\therefore$ & & $\begin{array}{l}24.03(22.41,25.55) \\
20.80 \\
20.24(19.34 .21 .4) \\
2.81\end{array}$ \\
\hline $\begin{array}{l}\text { Haandi Chenifie tal } \\
\text { Hamadi Chente tal }\end{array}$ & $\begin{array}{l}\substack{\text { Algeria } \\
\text { Algeria }} \\
\text { nat }\end{array}$ & 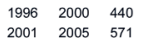 & $\because$ & & 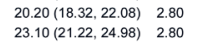 \\
\hline $\begin{array}{l}\text { Hamdichenifietal } \\
\text { Jedy-Anbae et al }\end{array}$ & 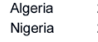 & 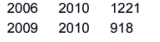 & & & 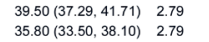 \\
\hline $\begin{array}{l}\text { Judy-Abata tal } \\
\text { Koiretal. }\end{array}$ & $\begin{array}{l}\begin{array}{l}\text { Niegria } \\
\text { Kenya }\end{array} \\
\text {. }\end{array}$ & $\begin{array}{lll}2009 & 21010 & 380 \\
2004 & 2008 & 1154\end{array}$ & - & & 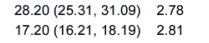 \\
\hline $\begin{array}{l}\text { Larvea a tal } \\
\text { Missauie tal. }\end{array}$ & $\begin{array}{l}\text { Shana } \\
\text { Tunisia }\end{array}$ & 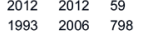 & & & 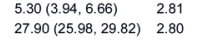 \\
\hline $\begin{array}{l}\text { Missaucital. } \\
\text { Missoui etal. }\end{array}$ & $\begin{array}{l}\text { Tusisia } \\
\text { Tunisia } \\
\text { The }\end{array}$ & 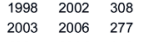 & & & 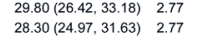 \\
\hline 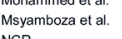 & 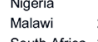 & 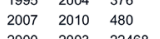 & $\bullet$ & & 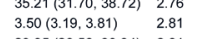 \\
\hline & $\begin{array}{l}\text { Sount Africa } \\
\text { south Africa }\end{array}$ & $\begin{array}{l}200020030322468 \\
2004 \\
2002 \\
20026\end{array}$ & & & 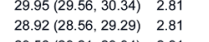 \\
\hline 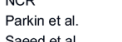 & $\begin{array}{l}\text { Soun Aluct } \\
\text { Uganda }\end{array}$ & 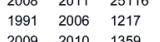 & - & & 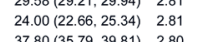 \\
\hline $\begin{array}{l}\text { Saghoke enal al. } \\
\text { Somdyyla et al. }\end{array}$ & $\begin{array}{l}\text { Suann } \\
\text { Gambia } \\
\text { South Africe }\end{array}$ & 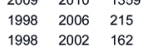 & & & 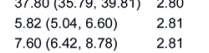 \\
\hline 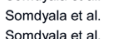 & $\begin{array}{l}\text { South Africa } \\
\text { Suric }\end{array}$ & $\begin{array}{lll}2003 & 2007 & 175\end{array}$ & & & 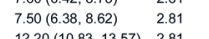 \\
\hline 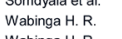 & 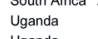 & 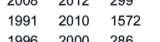 & + & & 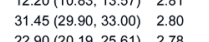 \\
\hline 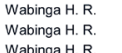 & 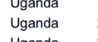 & 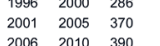 & & & $\begin{array}{lll}22.90(20.19,25.61) & 2.78 \\
29.60(26.52,32.68) & 2.78\end{array}$ \\
\hline $\begin{array}{l}\text { Wabinga H.R. } \\
\text { Sutroala (1-square }\end{array}$ & 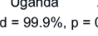 & & & & $\begin{array}{lll}31.202(28.04,434.36) & 2.77 \\
24,34(19.87,28.80) & 89.51\end{array}$ \\
\hline Moderate & & & & & \\
\hline $\begin{array}{l}\text { Dem etal. } \\
\text { Denewere tal. } \\
\text { topopotal. }\end{array}$ & 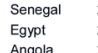 & 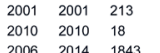 & 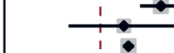 & & 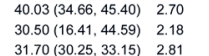 \\
\hline 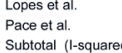 & $\begin{array}{l}\text { Angola } \\
\text { Rwanda } \\
\text { dw } 9.99 \% \text {. }\end{array}$ & 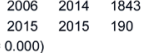 & - 1 & & $\begin{array}{ll}2.96(2.54 .3 .3 .38) & 2.81 \\
26.12(5.53,46.71) & 10.49\end{array}$ \\
\hline rall (1-squa & $.9 \%, \mathrm{p}$ & & $\infty$ & & $24.50(20.13,28.87) \quad 100.00$ \\
\hline E: Weights & rom ran & yis & & & \\
\hline
\end{tabular}

Figure 3. A. Pooled crude incidence rates of breast cancer in Africa by African region - population-based cancer registries. B. Pooled crude incidence rates of breast cancer in Africa by African region - population-based cancer registries (sensitivity analysis). 


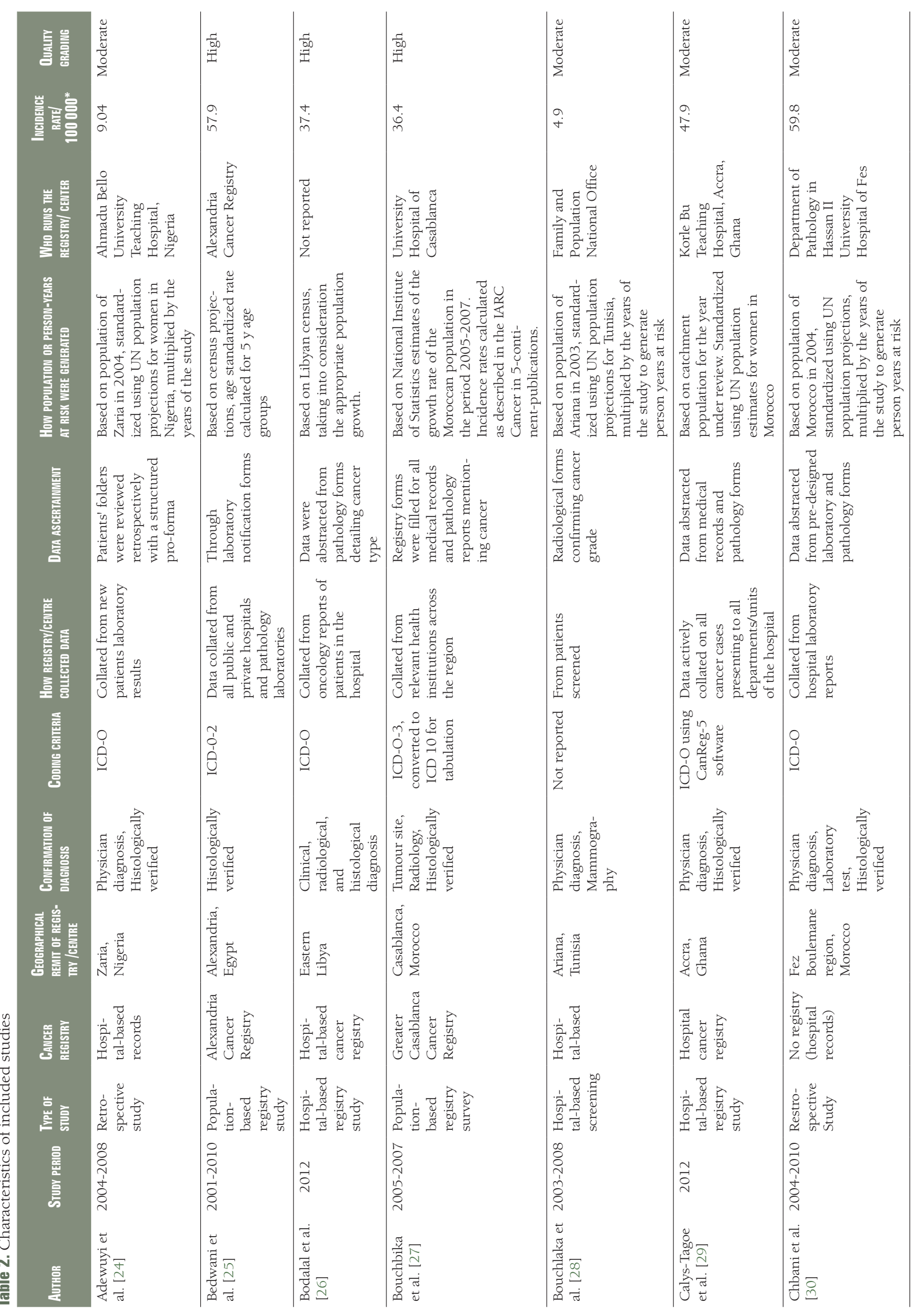




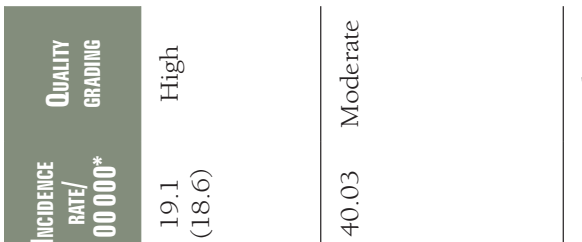

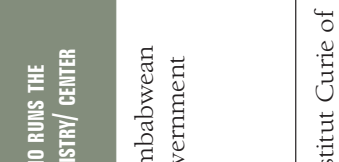

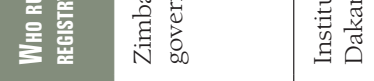

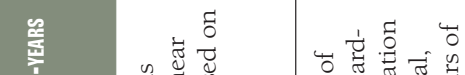

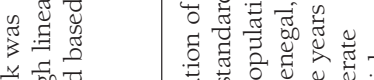

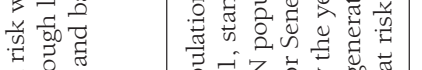

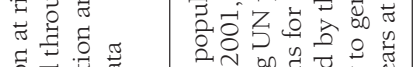

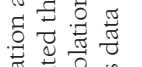

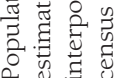

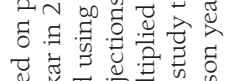

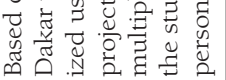

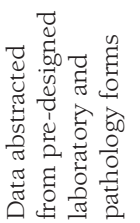

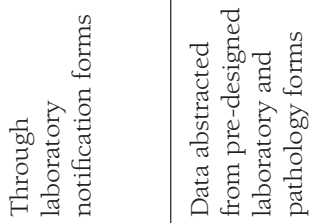

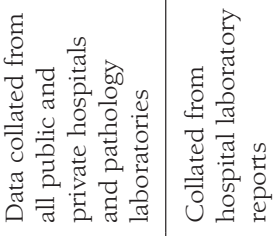

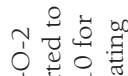

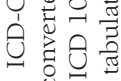

ํㅜㅇ

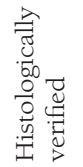

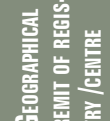

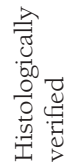

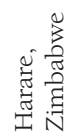

II

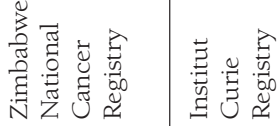

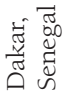

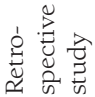

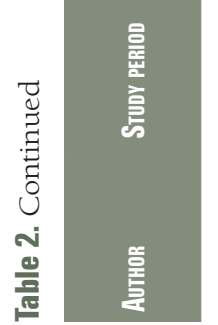

空

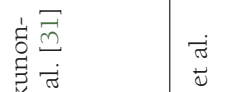

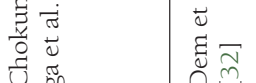

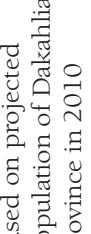

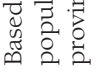

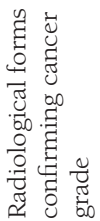

莕

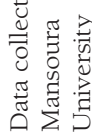

임

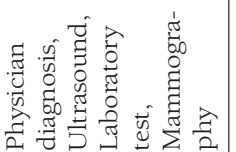

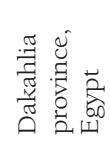

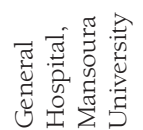

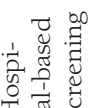

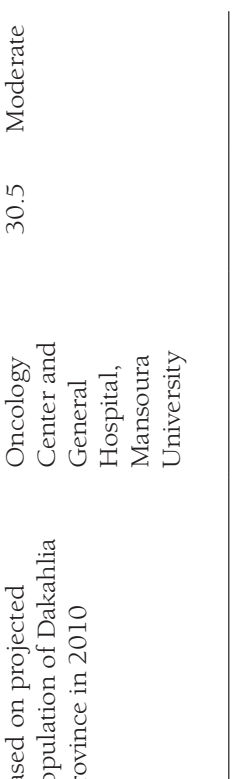

웅

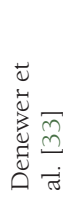

苞

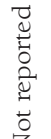

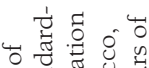

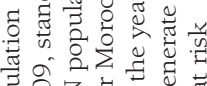

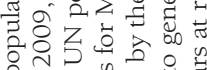

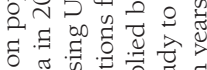

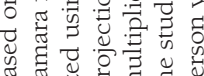

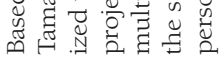

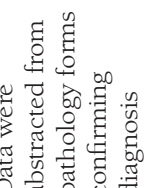

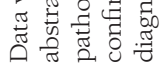

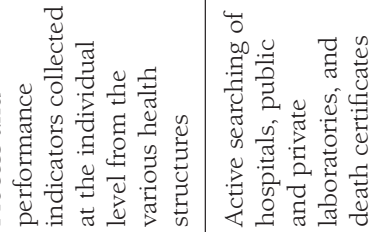

D

$\theta$

仓̊․

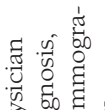

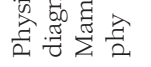

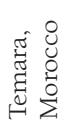

疍

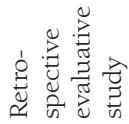

ᄋำ 궁

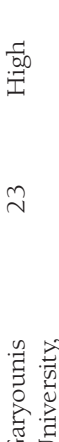

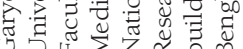

品

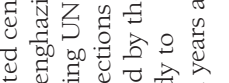

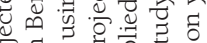

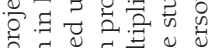

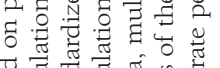

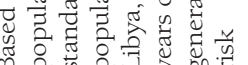

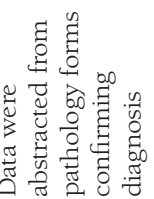

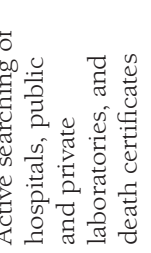

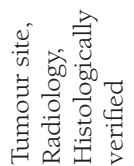

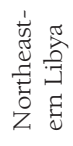

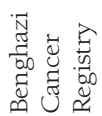

竞离

望

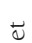

咅需 $\underset{\substack{5 \\ \infty}}{\infty}$

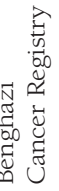

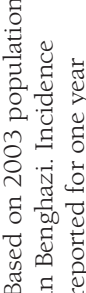

घี है

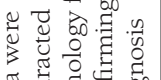

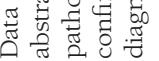

8

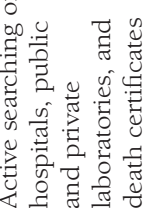

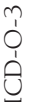

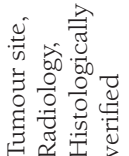

范

之ू

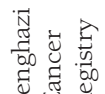
ตั

苟

ڤิ

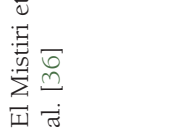




\begin{tabular}{|c|c|c|c|c|c|}
\hline 言害 & 点 & 离落 & 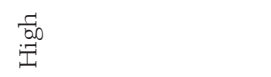 & $\begin{array}{l}\sqrt[5]{50} \\
: 3\end{array}$ & 离 \\
\hline & 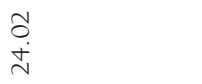 & ֶָ & & 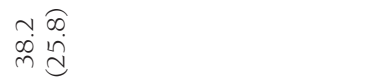 & $\stackrel{\text { I }}{\beth}$ \\
\hline 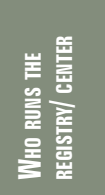 & 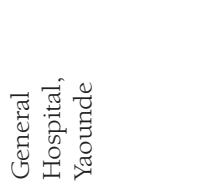 & 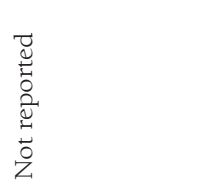 & 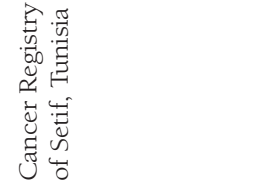 & 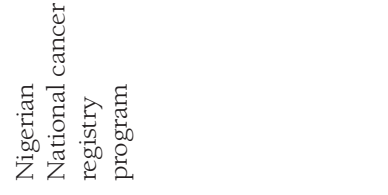 & 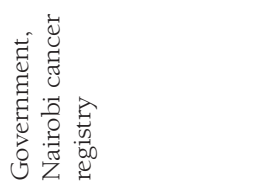 \\
\hline 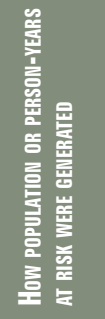 & 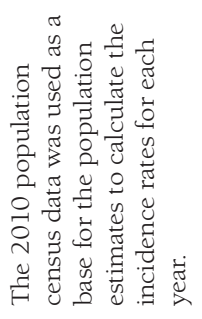 & 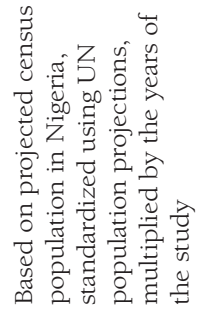 & 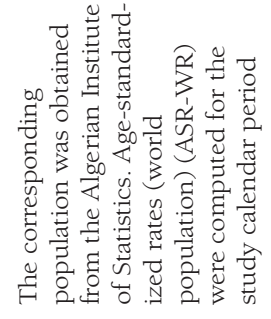 & 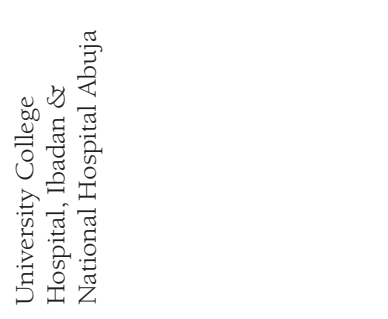 & 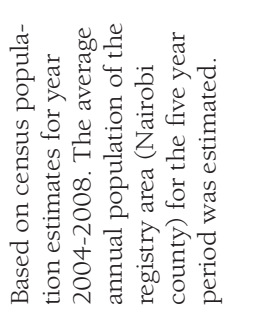 \\
\hline 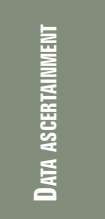 & 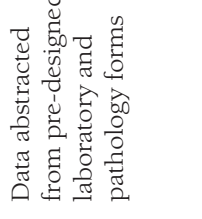 & 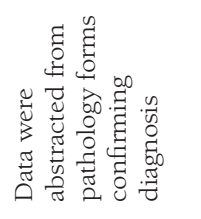 & 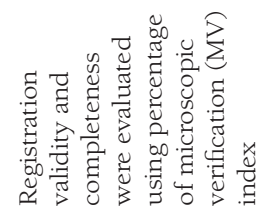 & 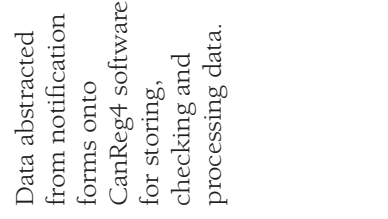 & 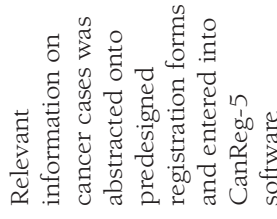 \\
\hline 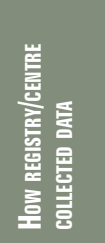 & 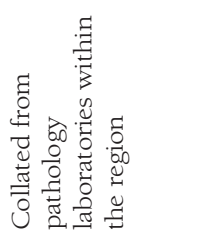 & 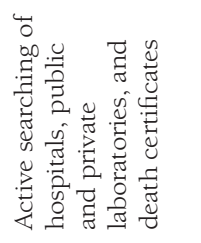 & 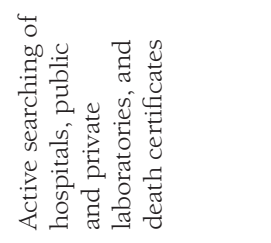 & 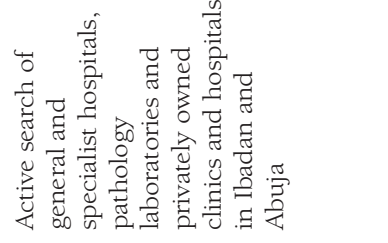 & 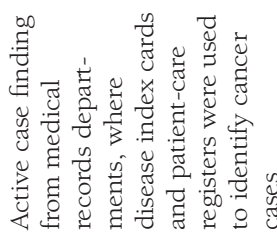 \\
\hline $\begin{array}{l}\text { 톨 } \\
\text { 产 } \\
\text { 言 }\end{array}$ & 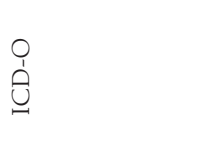 & $\begin{array}{l}\tilde{1} \\
\dot{1} \\
\stackrel{0}{0}\end{array}$ & $\begin{array}{l}0 \\
0 \\
0 \\
0 \\
0\end{array}$ & $\begin{array}{l}n \\
0 \\
\dot{1} \\
0\end{array}$ & 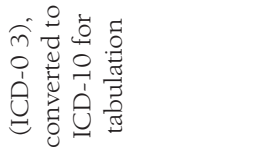 \\
\hline 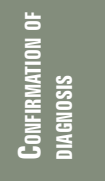 & 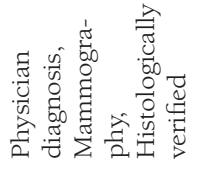 & 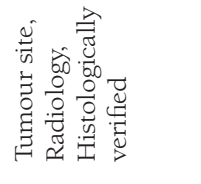 & 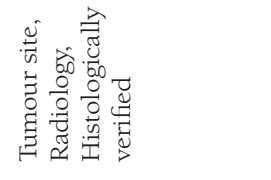 & 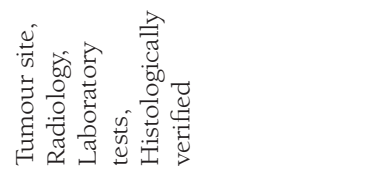 & 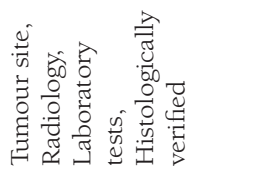 \\
\hline 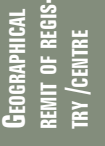 & 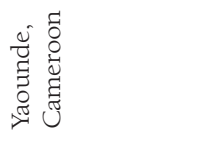 & $\begin{array}{l}\overrightarrow{D ̆}_{0} \\
\vec{z}\end{array}$ & 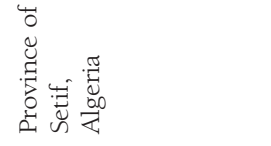 & 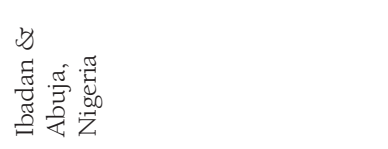 & 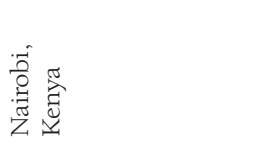 \\
\hline 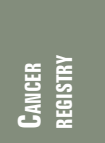 & 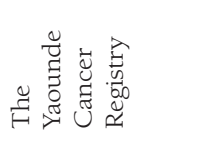 & 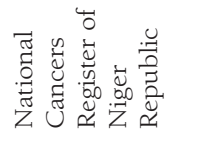 & 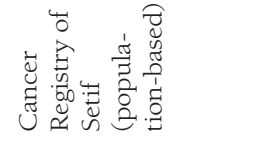 & 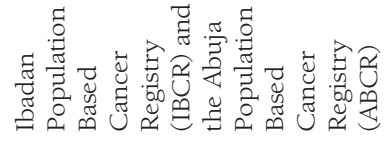 & 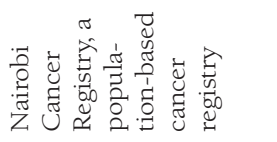 \\
\hline 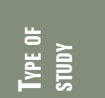 & 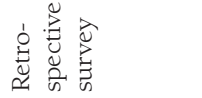 & 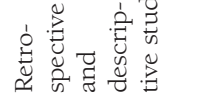 & 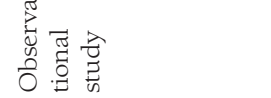 & 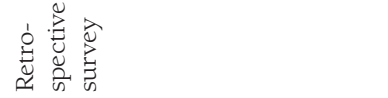 & 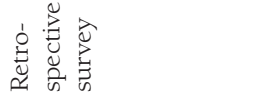 \\
\hline 言 & 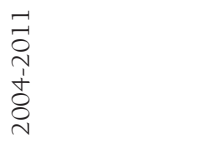 & 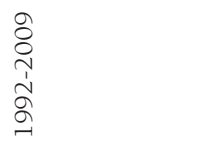 & 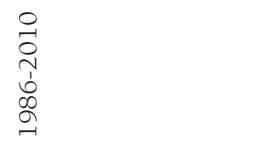 & 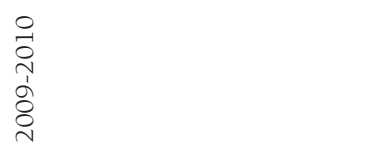 & 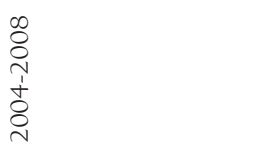 \\
\hline & 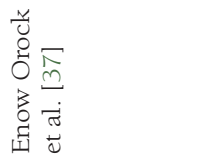 & 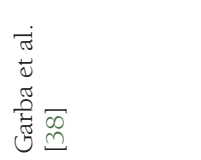 & 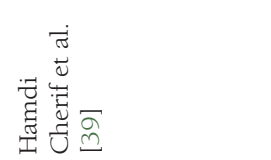 & 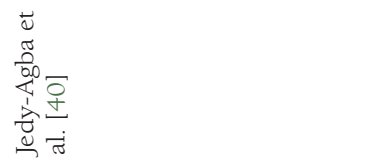 & 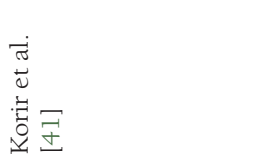 \\
\hline
\end{tabular}




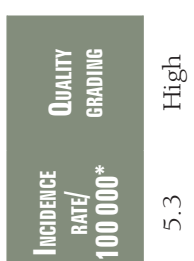

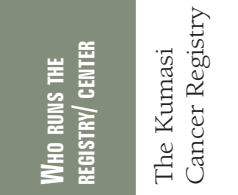

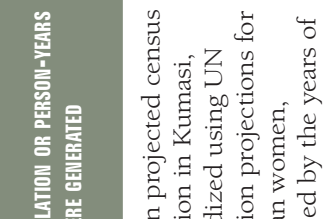

跣

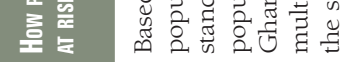

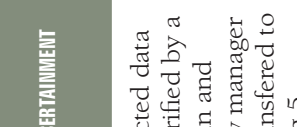

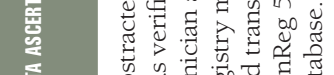

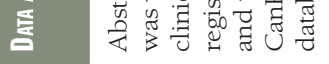

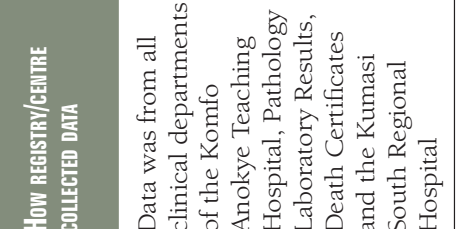

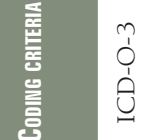

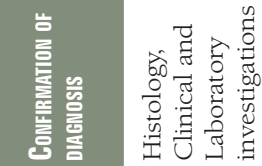

혈혔

咅咅言

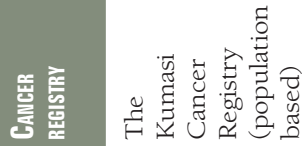

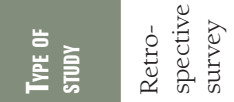

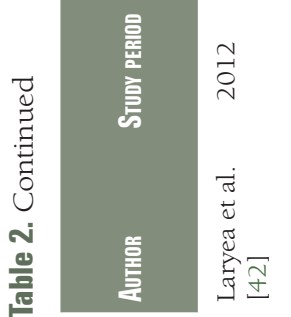

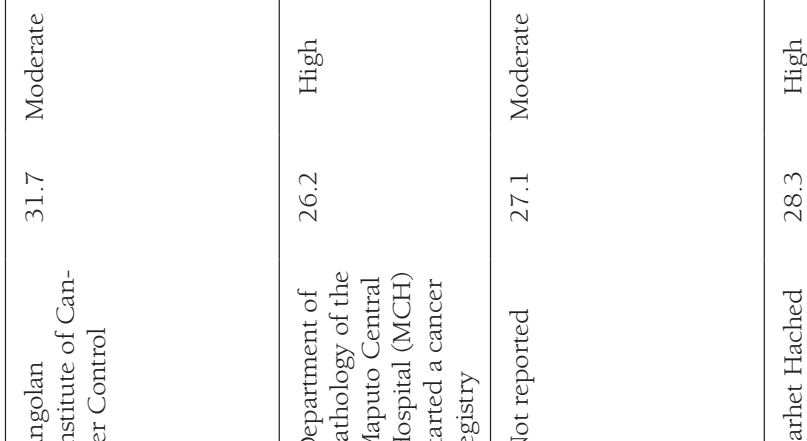

$\stackrel{5}{50}$

$\stackrel{m}{\infty}$

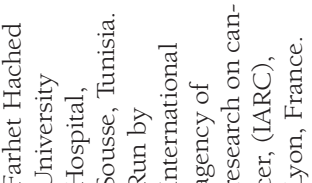

छี :

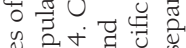

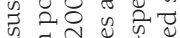

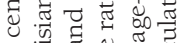

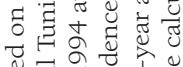

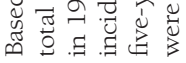

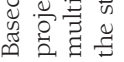

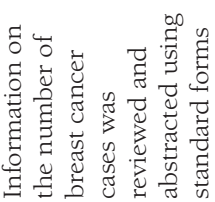

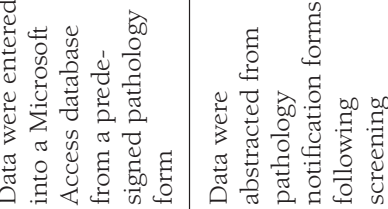

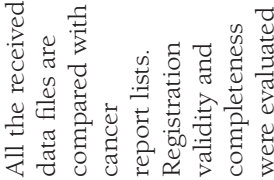

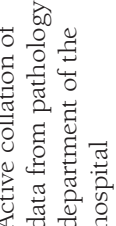

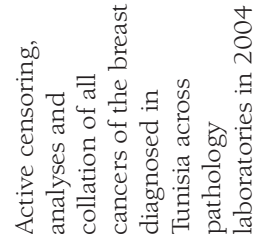

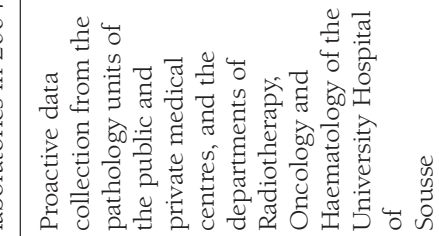

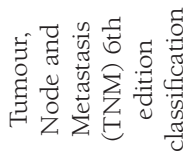

용

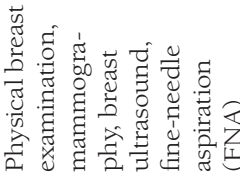

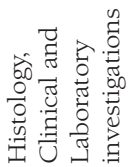

임

$\stackrel{1}{\circ}$

త్ర

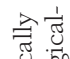

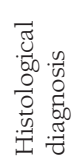

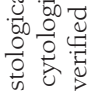

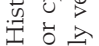

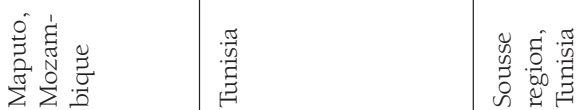

岁

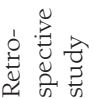

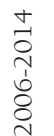

ल

๑)

站事

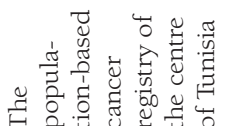

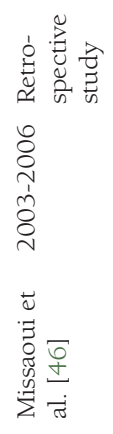




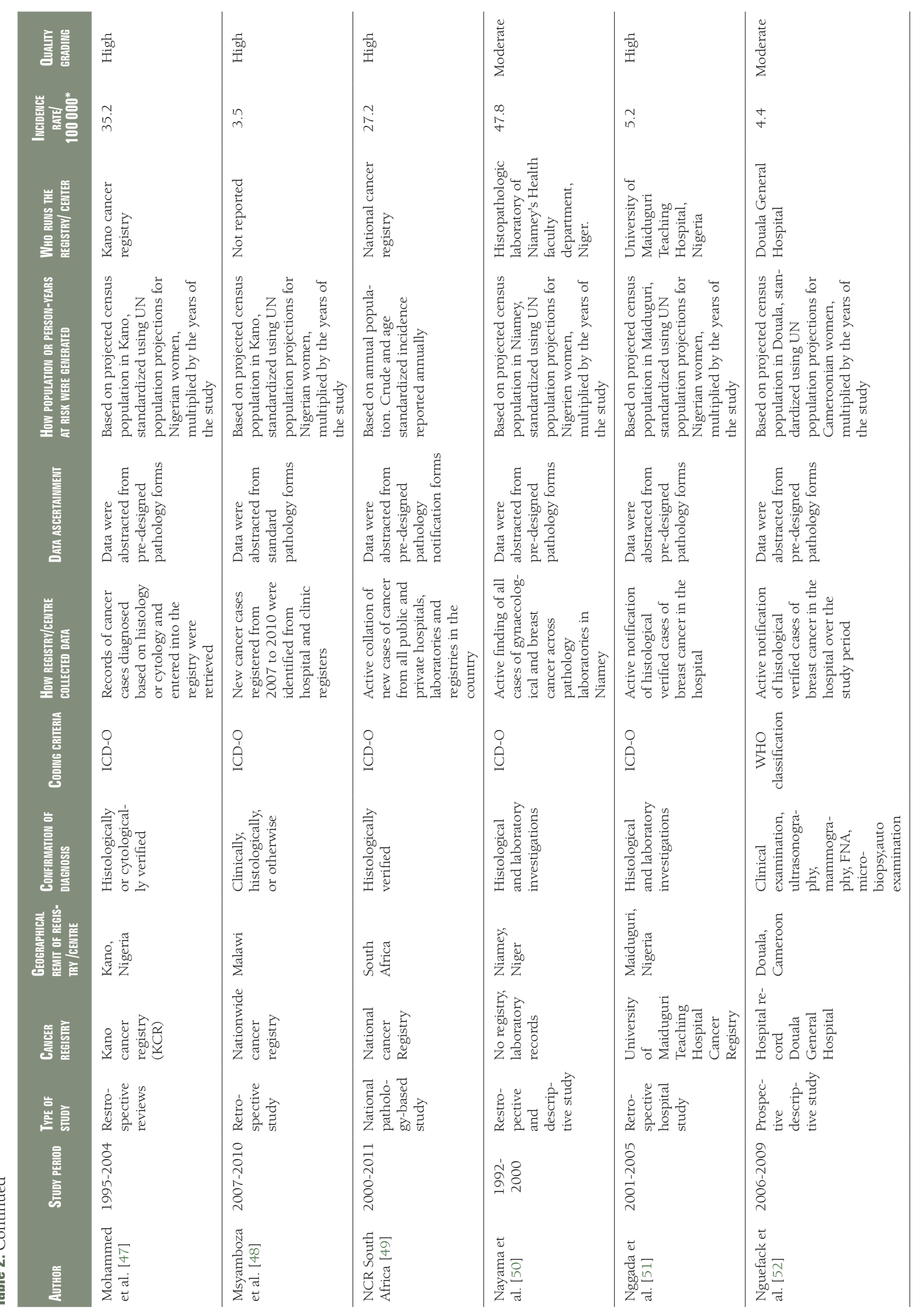



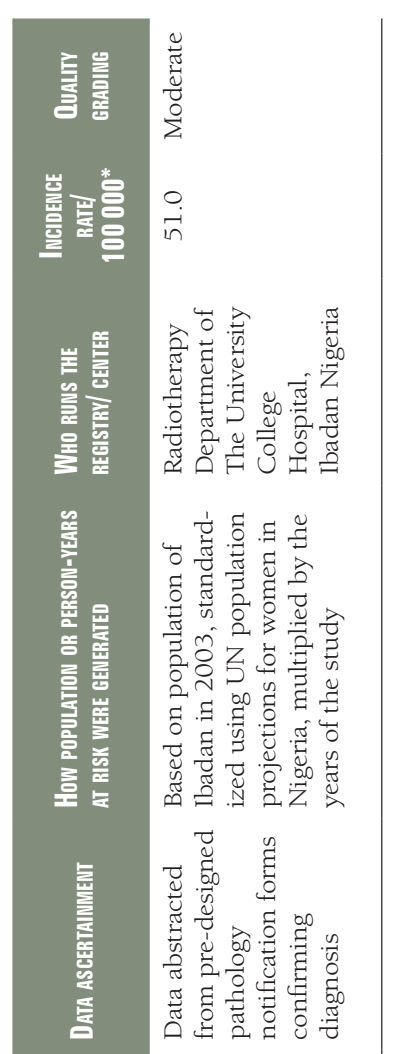

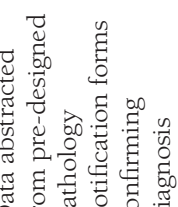

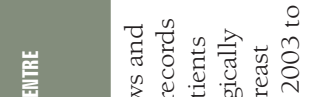

童高

異言

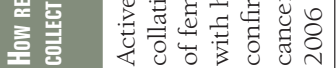

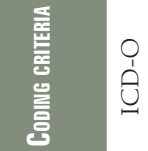

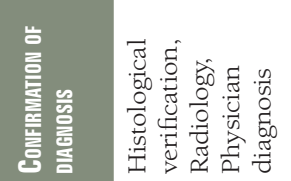

搐

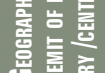

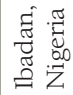

器产

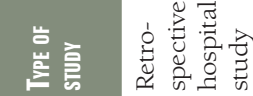

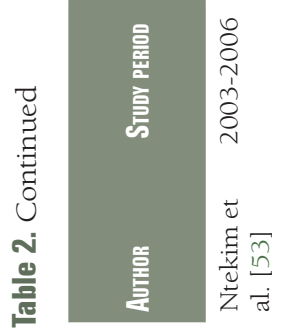

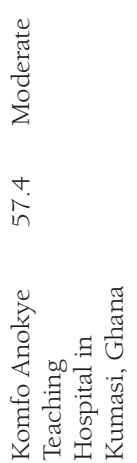

a to

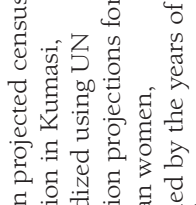

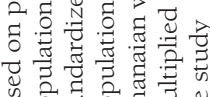

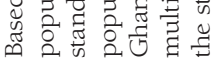

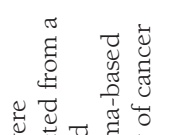

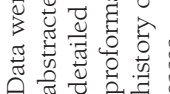

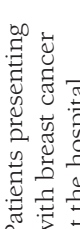

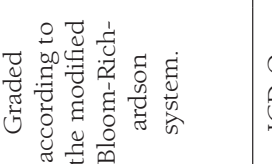

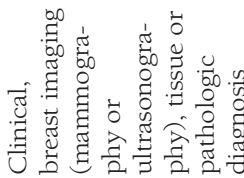

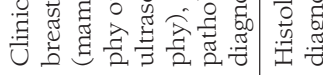

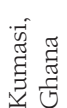

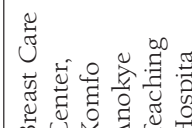

政

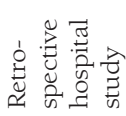

\&

年

에

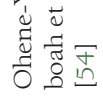

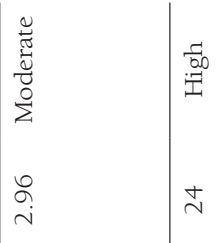

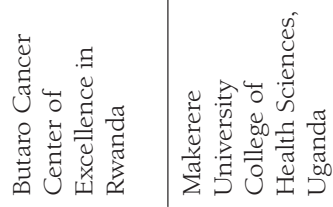

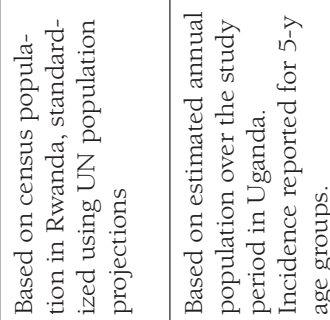

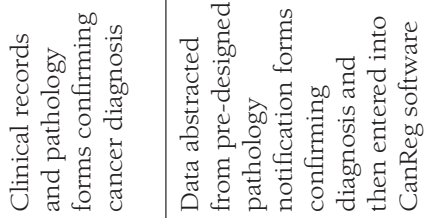

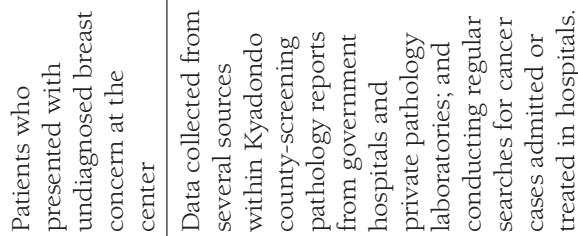

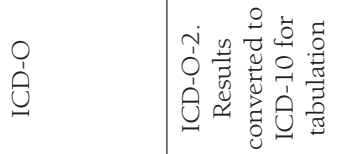

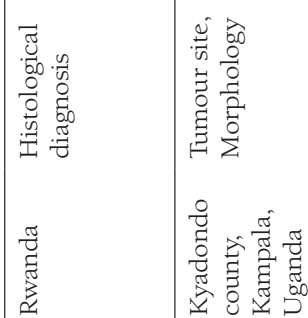

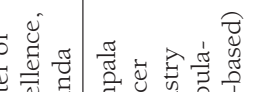

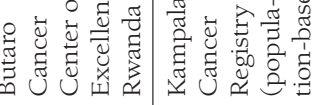

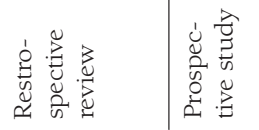

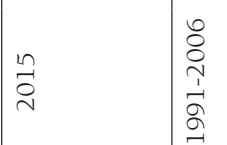

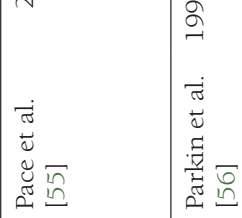

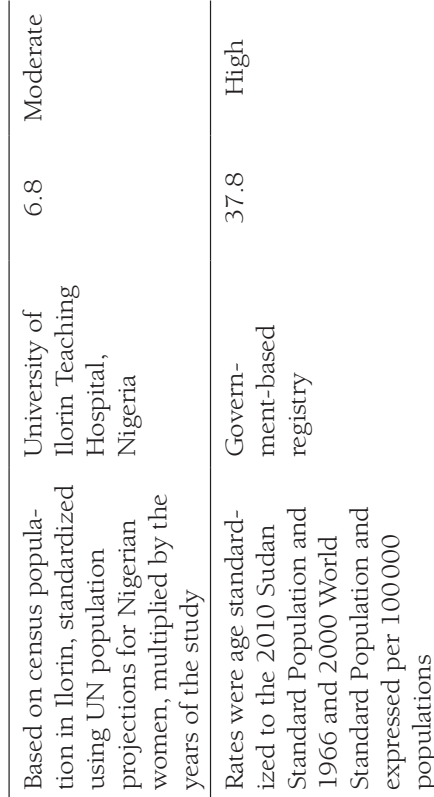

○ ซี I

要

3 远

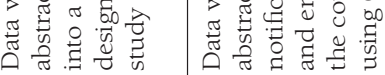

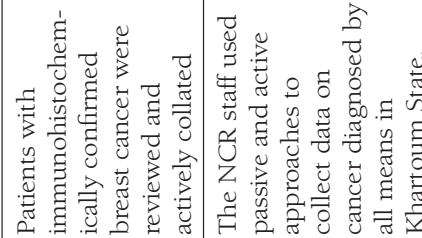

仓̊ำ

$\circ$

음

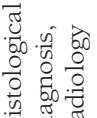

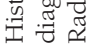

ह⿱士口心亍

.

它

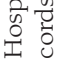

象

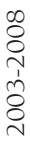

ㄴ.

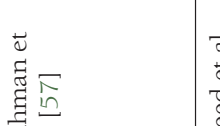




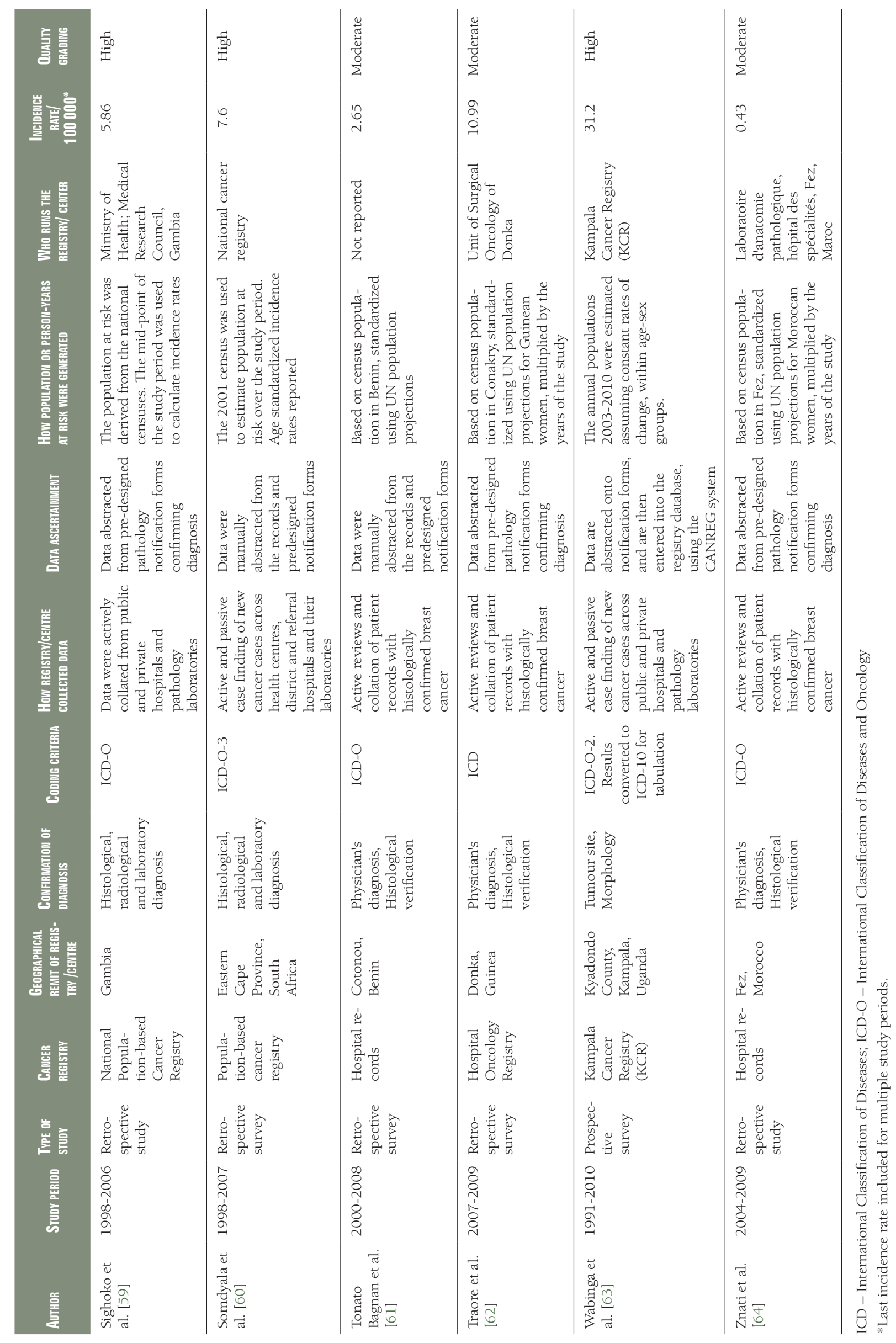


pooled incidence rate increased from 23.1 (95\% CI 18.7-27.5) to 26.3 (95\% CI 18.8-33.7) per 100 000 , respectively (Table 3). Further analysis by region revealed incidence of breast cancer in North Africa increased from 24.3 per 100000 (95\% CI 16.1-32.5) to 43.6 per 100000 (95\% CI 28.3-58.9) between 2000 and 2015. However, the incidence was about the same over the same period in SSA at 22.6 per 100000 (95\% CI 14.6-30.5) and 22.3 per 100000 (95\% CI 16.7 -27.9) (Table 3).

The meta-regression analysis showed a significant association of the crude incidence rates with age and year $(P=0.0012)$ (Table 4), which possibly demonstrates a relative consistency and representativeness of the data captured by population-based cancer registries. Assuming our pooled population-based registry estimates accounted for population growth, ageing and other demographic and epidemiological changes in Africa, these rates would account for 93890 (95\% CI 76007-111,774) breast cancer cases among women in 2000. This increased to 150526 breast cancer cases by 2015 (95\% CI 107600-192,880), based on the UN demographic projections for Africa.

\section{Hospital-based registries}

The results from the hospital-based registries (18 study sites) were slightly different (from the population-registries (Figure 4, plate A; Table 3). The overall pooled crude incidence rate was estimated at 23.6 per 100000 (95\% CI 18.5-28.7), with a median incidence of 15.0 per 100000 (IQR: 5.0-47.9). Further sensitivity analysis showed high quality studies had a pooled incidence rate at 17.4 per 100000 (95\% CI 2.2-32.5) compared to 25.4 per 100000 (95\% CI 19.8-31.0) for moderate quality hospital-based studies (Figure 4, plate B).

In contrast to the regional pattern observed in the population-based registries, SSA and Northern Africa had quite similar pooled rates at 24.0 per 100000 (95\% CI 17.5-30.4) and 23.2 per 100000 (95\% CI 6.6-39.7), respectively. Central Africa also had the lowest incidence rate at 4.4 per 100000 (95\% CI 3.15.7) (Figure 4, plate A). Although pooled incidence rates increased from 19.7 per 100000 (95\% CI 15.224.2) to 36.9 per 100000 (95\% CI 4.1-69.6) between 2000 and 2015, this increase in incidence rates was not associated with advancing age (Table 3). Further analysis by region over the same period (20002015) showed that North Africa and SSA markedly increased from 13.1 per 100000 (95\% CI 4.6-30.8) to 33.2 per 100000 (95\% CI 6.7-73.1), and 21.9 per 100000 (95\% CI 15.4-28.3) to 47.9 per 100000 (95\% CI 41.2-54.6), respectively. However, incidence rates decreased sharply between ages 30-39 years

Table 3. Sub-group meta-analysis of breast cancer crude incidence rates in Africa

\begin{tabular}{|c|c|c|c|c|c|c|c|}
\hline \multirow[b]{2}{*}{ Headings } & \multirow[b]{2}{*}{ Data } & \multicolumn{3}{|c|}{ Population-Based REgistRy } & \multicolumn{3}{|c|}{ HosPITAL-BASED REgISTRY } \\
\hline & & $\begin{array}{c}\text { Incidence per } 100000 \\
(95 \% \mathrm{CI})\end{array}$ & $I^{2}$ (P-value) & $\begin{array}{l}\text { Data } \\
\text { points }\end{array}$ & $\begin{array}{c}\text { Incidence per } 100000 \\
(95 \% \mathrm{CI})\end{array}$ & $\mathrm{I}^{2}$ (P-value) & $\begin{array}{c}\text { Data } \\
\text { points }\end{array}$ \\
\hline All & All & $\begin{array}{l}24.5 \text { (20.1-29.0); high } \\
\text { quality } 24.3(19.9-28.8)\end{array}$ & $99.9 \%(P<0.001)$ & 36 & $\begin{array}{l}23.6(18.5-28.7) ; \text { high } \\
\text { quality 17.4 (2.2-32.5) }\end{array}$ & $99.7 \%(P=<0.001)$ & 18 \\
\hline \multirow{6}{*}{$\begin{array}{l}\text { African } \\
\text { region }\end{array}$} & $\begin{array}{l}\text { Sub-Saharan } \\
\text { Africa (SSA) }\end{array}$ & $22.4(17.2-28.0)$ & $99.9 \%(P=<0.001)$ & 25 & $24.0(17.5-30.4)$ & $99.3 \%(P=<0.001)$ & 12 \\
\hline & Central Africa & $13.4(7.2-34.1)$ & $99.8 \%(P=<0.001)$ & 2 & $4.4(3.1-5.7)$ & - & 1 \\
\hline & East Africa & $28.0(21.7-33.7)$ & $98.8 \%(P=<0.001)$ & 7 & - & - & - \\
\hline & Southern Africa & $19.0(10.1-27.8)$ & $100 \%(P=<0.001)$ & 9 & $19.4(6.3-32.5)$ & $95.7 \%(P=<0.001)$ & 2 \\
\hline & West Africa & $24.2(15.4-33.0)$ & $99.6 \%(P=<0.001)$ & 7 & $27.4(18.6-36.2)$ & $99.4 \%(P=<0.001)$ & 9 \\
\hline & North Africa & $29.3(20.0-38.7)$ & $99.5 \%(P=<0.001)$ & 11 & $23.2(17.0-29.5)$ & $99.9 \%(P=<0.001)$ & 6 \\
\hline \multirow{4}{*}{ Age } & $30-39$ & $3.3(2.7-3.8)$ & $98.7 \%(P=<0.001)$ & 2 & $23.3(17.0-29.5)$ & $99.7 \%(P=<0.001)$ & 4 \\
\hline & $40-49$ & $22.4(13.1-31.7)$ & $99.6 \%(P=<0.001)$ & 7 & $7.1(4.6-9.6)$ & $91.3 \%(P=<0.001)$ & 5 \\
\hline & $50-59$ & $22.6(18.6-26.6)$ & $99.7 \%(P=<0.001)$ & 19 & $34.3(17.6-51.0)$ & $99.5 \%(P=<0.001)$ & 7 \\
\hline & $60+$ & $36.6(30.7-42.4)$ & $75.6 \%(P=0.043)$ & 8 & $27.1(7.3-47.0)$ & $97.9 \%(P=<0.001)$ & 2 \\
\hline \multirow{2}{*}{$\begin{array}{l}\text { Study } \\
\text { period* }\end{array}$} & $2000-2010$ & $\begin{array}{l}23.1 \text { (18.7-27.5); North } \\
\text { Africa } 24.3(16.1-32.5) \\
\text { SSA } 22.6(14.6-30.5)\end{array}$ & $99.7 \%(P=<0.001)$ & 20 & $\begin{array}{l}19.7 \text { (15.2-24.2); North } \\
\text { Africa 13.1 (4.6-30.8); } \\
\text { SSA 21.9 (15.4-28.3) }\end{array}$ & $99.6 \%(P=<0.001)$ & 14 \\
\hline & $2010-2015$ & $\begin{array}{l}26.3 \text { (18.8-33.7); North } \\
\text { Africa } 43.6(28.3-58.9) \\
\text { SSA } 22.3(16.7-27.9)\end{array}$ & $99.9 \%(P=<0.001)$ & 16 & $\begin{array}{l}36.9 \text { (4.1-69.6); North } \\
\text { Africa } 33.2 \text { (6.7-73.1); } \\
\text { SSA } 47.9 \text { (41.2-54.6) }\end{array}$ & $99.7 \%(P=<0.001)$ & 4 \\
\hline \multicolumn{2}{|c|}{ Median (Interquartile range) } & $28.1(18.6-31.3)$ & - & 36 & $15.0(5.0-47.9)$ & - & 18 \\
\hline
\end{tabular}


A.

Study Study

\begin{tabular}{|c|c|c|c|c|c|c|c|c|}
\hline Author & Country & starts & ends & Cases & & & Inc/100000 (95\% Cl) & Weight \\
\hline North & & & & & ! & & & \\
\hline Bodalal et al & Libya & 2012 & 2012 & 210 & 1 & $x$ & $37.40(32.34,42.46)$ & 5.41 \\
\hline Bouchlaka et al. & Tunisia & 2003 & 2008 & 40 & $\bullet$ & & $4.90(3.38,6.42)$ & 5.69 \\
\hline Chbani et al. & Morocco & 2004 & 2010 & 3424 & 1 & 2 & $59.80(57.20,62.40)$ & 5.63 \\
\hline El Fakir et al. & Morocco & 2009 & 2011 & 4 & 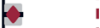 & & $2.50(0.05,4.95)$ & 5.64 \\
\hline Maalej et al. & Tunisia & 2004 & 2004 & 1408 & i & $\bullet$ & $34.13(32.34,35.92)$ & 5.68 \\
\hline Znati et al. & Morocco & 2004 & 2009 & 74 & 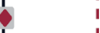 & & $0.43(0.33,0.53)$ & 5.72 \\
\hline \multicolumn{5}{|c|}{ Subtotal $(1-$ squared $=99.9 \%, p=0.000)$} & & & $23.15(6.57,39.72)$ & 33.77 \\
\hline & & & & & 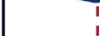 & & & \\
\hline West & & & & & 1 & & & \\
\hline Calys-Tagoe et al. & Ghana & 2012 & 2012 & 198 & i & - & $47.90(41.23,54.57)$ & 5.21 \\
\hline Nayama et al. & Niger & 1992 & 2000 & 168 & 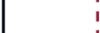 & - & $47.85(40.62,55.08)$ & 5.12 \\
\hline Ngadda et al. & Nigeria & 2001 & 2005 & 161 & $\Leftrightarrow$ & & $17.12(14.48,19.76)$ & 5.63 \\
\hline Ntekim et al. & Nigeria & 2003 & 2006 & 763 & 1 & $\rightarrow$ & $51.00(47.39,54.61)$ & 5.56 \\
\hline \multicolumn{2}{|c|}{ Ohene-Yeboah et al. Ghana } & 2004 & 2009 & 330 & i & & $57.41(51.22,63.60)$ & 5.27 \\
\hline Rahman et al. & Nigeria & 2003 & 2008 & 79 & $\nabla$ & & $6.76(5.29,8.23)$ & 5.69 \\
\hline \multicolumn{2}{|c|}{ Tonato Bagnan et al. Benin } & 2000 & 2008 & 93 & P & & $2.65(2.11,3.19)$ & 5.71 \\
\hline Traore et al. & Guinea & 2007 & 2009 & 178 & 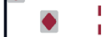 & & $11.00(9.37,12.63)$ & 5.68 \\
\hline Adewuyi et al. & Nigeria & 2004 & 2008 & 31 & $\Leftrightarrow$ & & $9.05(5.87,12.23)$ & 5.59 \\
\hline \multicolumn{5}{|c|}{ Subtotal $($ I-squared $=99.4 \%, p=0.000)$} & & & $27.39(18.58,36.19)$ & 49.47 \\
\hline . & & & & & i & & & \\
\hline South & & & & & i & & & \\
\hline Lorenzoni et al. & Mozambique & 1997 & 2002 & 64 & $\propto \vdots$ & & $12.80(9.68,15.92)$ & 5.60 \\
\hline Lorenzoni et al. & Mozambique & 2003 & 2008 & 132 & & & $26.20(21.73,30.67)$ & 5.48 \\
\hline \multicolumn{5}{|c|}{ Subtotal $(1-$ squared $=95.7 \%, p=0.000)$} & & & $19.40(6.27,32.53)$ & 11.07 \\
\hline . & & & & & I & & & \\
\hline Central & & & & & i & & & \\
\hline Nguefack et al. & Cameroon & 2006 & 2009 & 42 & $\bullet$ & & $4.40(3.07,5.73)$ & 5.69 \\
\hline \multicolumn{5}{|c|}{ Subtotal (1-squared $=. \%, p=)}$. & $\theta$ & & $4.40(3.07,5.73)$ & 5.69 \\
\hline \multicolumn{5}{|l|}{. } & & & & \\
\hline \multicolumn{5}{|c|}{ Overall $(I-$ squared $=99.7 \%, p=0.000)$} & & & $23.61(18.53,28.70)$ & 100.00 \\
\hline \multicolumn{5}{|c|}{ NOTE: Weights are from random effects analysis } & I & & & \\
\hline
\end{tabular}

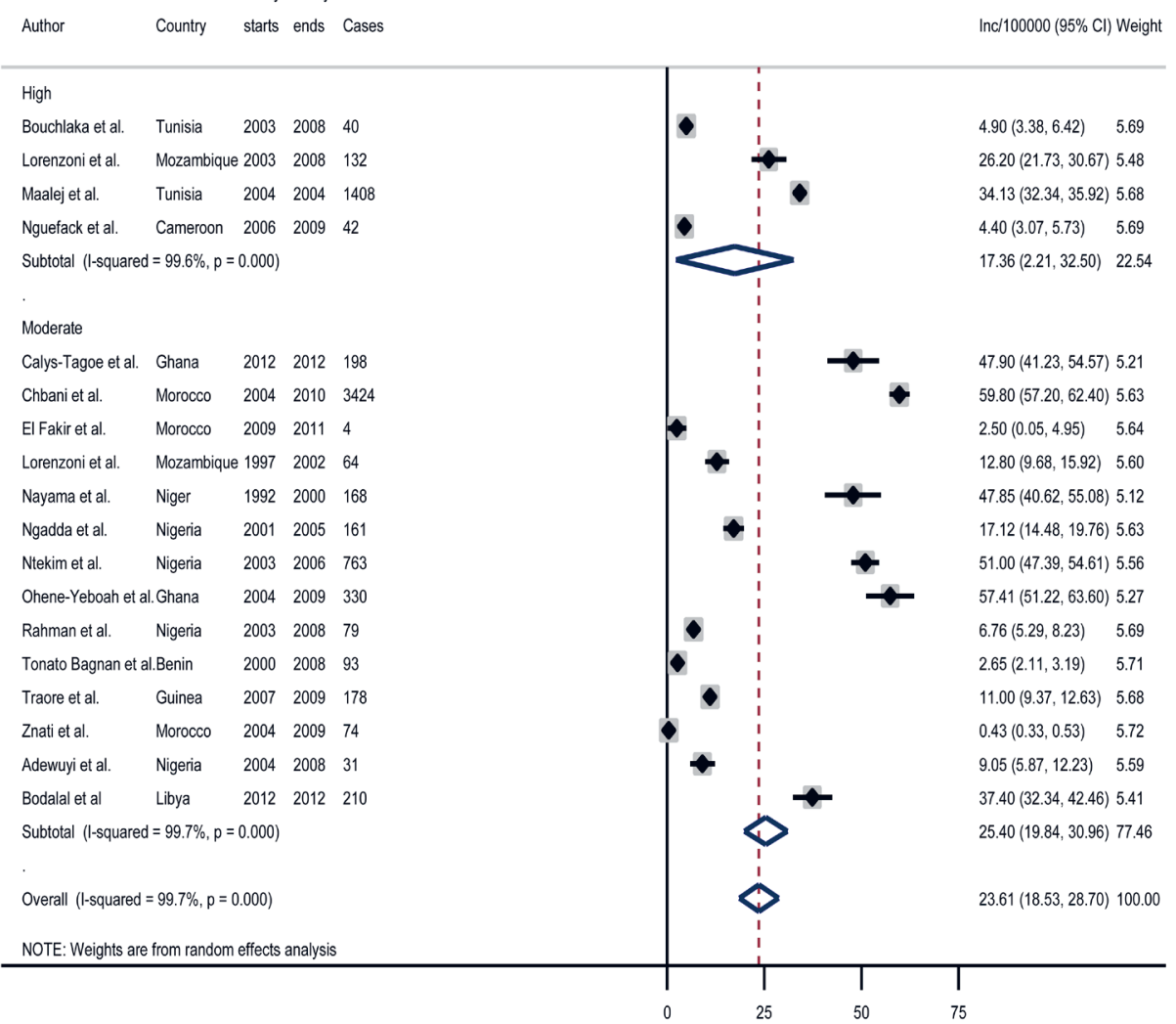

Figure 4. A. Pooled crude incidence rates of breast cancer in Africa by African region - hospital-based cancer registries. B. Pooled crude incidence rates of breast cancer in Africa by African region - population-based cancer registries (sensitivity analysis). 
Table 4. Meta-regression analysis by registry type

\begin{tabular}{|c|c|c|c|c|c|c|}
\hline Breast Cancer Incidence & CoEf. & StD. ERr. & $\mathbf{T}$ & $P>\mathrm{T}$ & UPPER CI & LOWER CI \\
\hline \multicolumn{7}{|l|}{ Population-based* } \\
\hline Age & 8.718566 & 2.188229 & 3.98 & $<0.001$ & 4.26658 & 13.17055 \\
\hline Year & 3.975006 & 3.495557 & 1.14 & 0.264 & -3.136759 & 11.08677 \\
\hline cons & 6.098437 & 4.850205 & 1.26 & 0.217 & -3.769378 & 15.96625 \\
\hline \multicolumn{7}{|l|}{ Hospital-based $\dagger$} \\
\hline Age & 1.865169 & 6.001334 & 0.31 & 0.760 & -10.92637 & 14.65671 \\
\hline Year & 14.49101 & 13.73779 & 1.05 & 0.308 & -14.79038 & 43.77241 \\
\hline _cons & -10.83332 & 27.9259 & -0.39 & 0.704 & -70.35596 & 48.68932 \\
\hline
\end{tabular}

$\mathrm{CI}$ - confidence interval

*Number of observations $=36$. REML estimate of between-study variance $(\operatorname{tau} 2)=105.9 . \%$ residual variation due to heterogeneity (I-squared_res) $=99.56 \%$. Proportion of between-study variance explained (Adj R-squared) $=29.96 \%$. Joint test for all covariates Model $F(2,33)=8.34$. With Knapp-Hartung modification Prob $>\mathrm{F}=0.0012$.

Number of observations $=18$

$\dagger$ REML estimate of between-study variance $(\operatorname{tau} 2)=443.7$. \% residual variation due to heterogeneity (I-squared_res) $=99.43 \%$. Proportion of between-study variance explained (Adj R-squared) $=0.09 \%$. Joint test for all covariates Model $F(2,15)=1.00$. With Knapp-Hartung modification Prob>F $=0.3926$.

and 40-49 years from 23.3 (95\% CI 17.0-29.5) to 7.1 (95\% CI 4.6-9.6) per 100000; peaked at 50-59 years (34.3 per $100000,95 \%$ CI 17.6-51.0), but dropped again at age 60 years and above to 27.1 per 100000 (95\% CI 7.3-47.0) (Table 3). The meta-regression showed no significant relationship between incidence, age and years covered $(P=0.3926)$ (Table 4), which perhaps reflects incomplete data capture in the hospital registries (no data from East Africa), and is probably partly due to our inability to appropriately define the catchment area of the hospital.

\section{DISCUSSION}

As the leading cause of cancer globally among women, breast cancer has continued to attract interest among experts and clinicians, particularly towards appropriately quantifying its burden and identifying risks across countries. In Africa, experts have continued to work towards addressing several challenges in the response to a growing cancer burden [14]. However, with relatively scant, incomplete, or poorly representative data on cancer in many settings, it remains difficult to effectively address this burden [15]. This study provides the first systematic review and meta-analysis of publicly available evidence on the incidence of female breast cancer in Africa with a view to improving the understanding of the epidemiology of the disease in the region. This endeavor yielded some findings.

Our study showed that population-based cancer registries remain the main sources of data on breast cancer in Africa as these provided $67 \%$ of data in this study. Current data from most hospital-based cancer registries are rather too incomplete and inconsistent to be deployed in the understanding of cancer epidemiology in Africa [12], as evidenced from the quality assessment. In the population-based registries, we observed that the estimated regional variation in breast cancer incidence in Africa was consistent with the GLOBOCAN 2008 and 2012 studies, with the incidence in North Africa higher than observed in SSA $[6,9]$. The estimated incidence of breast cancer in North Africa (29.3 per 100 000) was almost same as values reported by the global burden of diseases (GBD) collaborators for the region (29.7 per 100000 ) [2]. However, the GBD collaborators noted a relatively higher incidence rate in SSA in 2015 at 34.1 per 100000 compared to our estimate of 22.4 per 100000 (95\% CI 17.2-28.0) for SSA [2]. The GBD estimates are thus suggestive of a higher incidence of breast cancer in SSA compared to North Africa, which are in contrast to our findings of the opposite, and possibly raises potential questions regarding the rising trend of breast cancer in SSA compared to North Africa. The differences may be partly attributable to sources and completeness of data, as well as modeling approaches employed in the GBD estimates compared to the present study. Although some previous reports have shown that the estimated incidence rates of breast cancer across North African states are higher than in SSA, and comparable to rates in some high-income settings [65-67]. The regional differences in Africa may be indicative of racial and ethnic disparities in invasive breast cancer epidemiology in the region, as already observed in the variations in incidence and mortality patterns between black and white women globally $[68,69]$. Dickens and colleagues noted that the clinical spectrum of breast cancer in Africa is highly heterogenous across population groups [70], with this often linked to variations in age at diagnosis, staging, time trends, and genet- 
ic and environmental risks $[69,71]$. Moreover, the different stages of fertility transition currently observed in Africa as evidenced by declining births and widening birth intervals across population groups may also be an important driver of the regional differences [71].

Across both population- and hospital-based registries, we reported lower incidence rates in Central Africa. However, these estimates have wide uncertainty intervals, possibly reflecting the limited data in the region, and the substantial variation and imprecision in reported figures already noted across African countries $[10,72]$. Some authors have reported the lack of data in Central Africa as a major factor mitigating against the estimation of disease burden and ensuring evidence-based interventions in the region $[12,73,74]$. Balekouzou and colleagues specifically noted that the epidemiology of breast cancer in Central Africa remain poorly understood, owing to sparse data on cancer in the region [75]. Ongoing civil unrest in some parts of Central Africa influencing the capacity of the health systems to adequately capture cancer cases may have resulted in a relatively low cancer ascertainment.

The mean age of populations covered in both the population- and hospital-based registries ranged from 30.6 to 60.8 years (median 50.2 years), with over $33 \%$ and $81 \%$ of population in ages $30-49$ years, and 30-59 years, respectively. This, albeit subject to further validations, may be suggestive of a high incidence of breast cancer among younger age groups in Africa. For example, Jedy-Agba and colleagues reported that many cases of breast cancer in SSA were diagnosed in the ages 35-49 years, with many presenting at advanced late-stage cancer [7]. Some authors have argued that the higher proportion of younger women in Africa, due to a relatively lower life expectancy on the continent, may have been responsible for the increasing breast cancer incidence reported among younger age groups [14,76]. However, epidemiological transitions, rapid urbanization with increased adoption of unhealthy lifestyles, increasing prevalence of obesity in younger populations, changing reproductive behaviours, including early menarche, low parity, advanced age at first pregnancy, and low self-breast examination for breast cancer among adolescents and young women have been identified as possible risk factors [14,76,77].

The poor state of many health systems in Africa and their declining capacity to lead cancer preventive initiatives and response to overall health needs of the population, as compared to developed countries, are also major concerns. Abdulrahman and Rahman reported that there are significant differences in age and stage at presentation between women in Africa and Europe, with more than half of breast cancer patients in Nigeria and Libya presenting at relatively younger age, mostly with advanced stage III or IV disease [78]. Parkin et al. equally noted that the lifetime risk of dying from breast cancer in young African women is about twice the risk in high-income countries [56].

Meanwhile, we observed some important variations in the estimates reported from population-and hospital-based registries in this study. Although our estimated incidence of breast cancer increased between 2000 and 2015 in both registries, suggestive of a rising breast cancer incidence in Africa, there was however a more significant rise in breast cancer incidence with advancing age in the population-based registries. This was clearly different from our finding from the hospital-based studies. Moreover, from population-based registries, the breast cancer incidence in North Africa (29.7 per 100 000) was higher than the incidence in SSA (22.4 per 100000), while hospital-based registries provided relatively similar estimates (23.2 per 100000 vs 24.0 per 100000). These variations, notwithstanding our estimation approach, further raise concerns on the completeness and representativeness of data in African registries. Population-based cancer registries have been widely regarded as being the gold standard for accurate data collation on cancer [79], as they cover wide geographical areas including communities, and employ standard coding methods and estimation of person-years [12]. However, challenges have been identified in the establishment and effective functioning of population-based registries across Africa due to poor funding, lack of skilled personnel, and low priorities from many governments, with this affecting the estimates provided $[12,79]$. Some authors have reported that hospital-based registries can indeed complement the few population-based registries, particularly by providing data on health service provision and access, which is useful for appropriate planning and public health response [79]. To address challenges with data consistency and representativeness, efforts may also be directed at improving geographical mapping of hospitals to appropriately determine the catchment area or population covered, and generate standardized methods to calculate cancer incidence that are representative of the population [12].

The application of precision medicine across world regions is still evolving [3]. However, translating this into prevention and management of breast cancer in Africa also requires additional efforts with cancer registration [3]. It is important for future research to decipher if the advanced stage of breast cancer presentation in Africa could be linked to unique aggressive biological characteristics of the malignancy, or 
some specific intrinsic host factors in African populations $[7,76]$. This is essential to guide health care practitioners to adequately respond to known and prevailing health disparities with respect to genetic determinants, predisposition and susceptibility to breast cancer across different ethnic groups on the continent [3]. However, the research capacity in this regard is relatively poor, and additionally limited by the lack of comprehensive and nationally representative data on breast cancer across African countries [5]. Hence, public health promoting behaviors that decrease breast cancer incidence still appears be most important and feasible preventive strategy across Africa.

The limitations of this study are also related to our key findings. First, though we performed a compressive data retrieval process, the empirical estimates we provided are limited by the absence of population-based cancer registries in many African countries (only 22 African countries represented), which has implications for the precision and representativeness of our estimates. Although, about $67 \%$ of studies were conducted in population-based cancer registries, many of these registries only covered subnational populations. As highlighted in the results, Nigeria and South Africa had the highest data points in this study. However, of all entries, South Africa particularly provided a complete national registry report of histologically-diagnosed breast cancer cases covering a period of 18 years, although only recent reports suggested an upgrade to population-based cancer registration system [80]. As noted, across data from hospital-based reports, we relied on the UN demographics for the country to estimate reference populations and person-years when they were not provided. This could have led to a relative under-estimation of cancer incidence due to resultant large denominators derived from the UN demographics. Besides, estimates were not age-standardized which limits direct comparisons with other world regions. Moreover, the heterogeneity across selected studies was high $\left(\mathrm{I}^{2}>90 \%\right)$, which further reflects the need to address varying study designs, case ascertainments, referral approaches, and standards for collation of cancer data across different population groups, as these are important sources of heterogeneity. Despite these limitations, it is still important to present to the public the challenges arising from data availability on breast cancer research in Africa, the varying estimates available, and the implication of this on the response to a rising burden of breast cancer on the continent.

\section{CONCLUSIONS}

The efforts of various researchers and organizations, particularly the IARC, United States National Cancer Institute (NCI) and African Organization for Research and Training in Cancer (AORTIC), must be acknowledged in the response to cancer in Africa. Data availability remains an ongoing limitation in understanding and estimating the incidence of breast cancer on the continent. Further issues from available data include inconsistency in data collation and quality across existing cancer registries. However, available evidence is suggestive of a growing incidence of breast cancer in Africa. With population growth and ageing, exposures to known risks, and relative weak health systems in Africa, it is unlikely this trend will change in the short-term in the absence of effective interventions, with this further straining the health system in many African countries. As various funders and institutions develop their focuses, we assert that strategies for controlling and treating breast cancer patients must include activities that support active and comprehensive data collation and cancer registration (population- and hospital-based) on the African continent, and more emphasis on the biological and histological characteristics of breast cancer cells across different population groups.

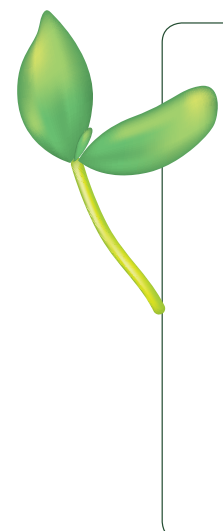

Acknowledgements: The authors wish to thank Prof. Clement Adebamowo, Prof. Emeka Iweala and Dr Suhail Doi for the intellectual support in the conduct of this study.

Funding: None

Authorship declaration: DA conceived the study. OYS and AA conducted the data extraction with oversight from DA. DA and MOH conducted the analysis. DA drafted the paper. DA, WJ, RAD, AAA, AOA, SM, MG, AA, $\mathrm{MOH}$ and KYC contributed to the final writing of the paper and checked for important intellectual content. All authors approved the final draft of the manuscript.

Conflict of interest: DA and KYC are members of the editorial board of the Journal of Global Health. Authors have completed the Unified Competing Interest form at www.icmje.org/coi_disclosure.pdf (available on request from the corresponding author). None of the participating authors has a conflicting financial or other interest related to the work detailed in this manuscript. 
1 GBD 2015 Mortality and Causes of Death Collaborators. Global, regional, and national life expectancy, all-cause mortality, and cause-specific mortality for 249 causes of death, 1980-2015: a systematic analysis for the Global Burden of Disease Study 2015. Lancet. 2016;388:1459-544. Medline:27733281 doi:10.1016/S0140-6736(16)31012-1

2 Global Burden of Disease Cancer Collaboration; Fitzmaurice C, Allen C, Barber RM, Barregard L, Bhutta ZA, et al. Global, Regional, and National Cancer Incidence, Mortality, Years of Life Lost, Years Lived with Disability, and Disability-Adjusted Life-years for 32 Cancer Groups, 1990 to 2015: A systematic analysis for the Global Burden of Disease Study. JAMA Oncol. 2017;3:524-48. Medline:27918777 doi:10.1001/jamaoncol.2016.5688

3 Silverstein A, Sood R, Costas-Chavarri A. Breast cancer in Africa: Limitations and opportunities for application of genomic medicine. Int J Breast Cancer. 2016;2016:4792865. Medline:27413551 doi:10.1155/2016/4792865

4 Brinton L, Figueroa J, Adjei E, Ansong D, Biritwum R, Edusei L, et al. Factors contributing to delays in diagnosis of breast cancers in Ghana, West Africa. Breast Cancer Res Treat. 2017;162:105-14. Medline:28025716 doi:10.1007/ s10549-016-4088-1

5 Pace LE, Shulman LN. Breast cancer in Sub-Saharan Africa: Challenges and opportunities to reduce mortality. Oncologist. 2016;21:739-44. Medline:27091419 doi:10.1634/theoncologist.2015-0429

6 Ferlay J, Soerjomataram I, Dikshit R, Eser S, Mathers C, Rebelo M, et al. Cancer incidence and mortality worldwide: sources, methods and major patterns in GLOBOCAN 2012. Int J Cancer. 2015;136:E359-86. Medline:25220842 doi:10.1002/ijc.29210

7 Jedy-Agba E, McCormack V, Adebamowo C, dos-Santos-Silva I. Stage at diagnosis of breast cancer in sub-Saharan Africa: a systematic review and meta-analysis. Lancet Glob Health. 2016;4:e923-35. Medline:27855871 doi:10.1016/ S2214-109X(16)30259-5

8 al-Haddad BJ, Jedy-Agba E, Oga E, Ezeome ER, Obiorah CC, Okobia M, et al. Comparability, diagnostic validity and completeness of Nigerian cancer registries. Cancer Epidemiol. 2015;39:456-64. Medline:25863982 doi:10.1016/j. canep.2015.03.010

9 Ferlay J, Shin HR, Bray F, Forman D, Mathers C, Parkin DM. Estimates of worldwide burden of cancer in 2008: GLOBOCAN 2008. Int J Cancer. 2010;127:2893-917. Medline:21351269 doi:10.1002/ijc.25516

10 Parkin DM, Wabinga H, Nambooze S. Completeness in an African cancer registry. Cancer Causes Control. 2001;12:14752. Medline:11246843 doi:10.1023/A:1008966225984

11 Brinton LA, Awuah B, Nat Clegg-Lamptey J, Wiafe-Addai B, Ansong D, Nyarko KM, et al. Design considerations for identifying breast cancer risk factors in a population-based study in Africa. Int J Cancer. 2017;140:2667-77. Medline:28295287 doi:10.1002/ijc.30688

12 Gakunga R, Parkin DM. African Cancer Registry N. Cancer registries in Africa 2014: A survey of operational features and uses in cancer control planning. Int J Cancer. 2015;137:2045-52. Medline:26135162 doi:10.1002/ijc.29668

13 Adeloye D, Grant L. A Review of Cancers in Africa. In: Chronic Non-Communicable Diseases in Low- and Middle-Income Countries. Wallingford, Oxfordshire; Boston, MA: CABI; 2016.

14 Brinton LA, Figueroa JD, Awuah B, Yarney J, Wiafe S, Wood SN, et al. Breast cancer in Sub-Saharan Africa: opportunities for prevention. Breast Cancer Res Treat. 2014;144:467-78. Medline:24604092 doi:10.1007/s10549-014-2868-z

15 Jemal A, Bray F, Forman D, O’Brien M, Ferlay J, Center M, et al. Cancer burden in Africa and opportunities for prevention. Cancer. 2012;118:4372-84. Medline:22252462 doi:10.1002/cncr.27410

16 Parkin DM, Bray F, Ferlay J, Pisani P. Estimating the world cancer burden: Globocan 2000. Int J Cancer. 2001;94:1536. Medline:11668491 doi:10.1002/ijc. 1440

17 Bray F, Ferlay J, Laversanne M, Brewster DH, Mbalawa CG, Kohler B, et al. Cancer Incidence in Five Continents: Inclusion criteria, highlights from Volume X and the global status of cancer registration. Int J Cancer. 2015;137:2060-71. Medline:26135522 doi:10.1002/ijc.29670

18 International Agency for Research on Cancer. Cancer in Africa: epidemiology and prevention. IARC Sci Publ. 2003;•・•:1414. Medline:14570050

19 World Bank. World Bank list of economies. 2013. Available: http://data.worldbank.org/about/country-classifications/ country-and-lending-groups. Accessed: 7 January 2014.

20 World Health Organization. International classification of diseases for oncology (1st ed). Geneva: World Health Organization; 1976.

21 International Agency for Research on Cancer. International classification of diseases for oncology: morphology. Bethesda MD: National Cancer Institute; 1987

22 Percy CL, Van Holten V, Muir CS. International classification of diseases for oncology (2nd ed). Geneva: World Health Organization; 1990

23 DerSimonian R, Laird N. Meta-Analysis in Clinical Trials. Control Clin Trials. 1986;7:177-88. Medline:3802833 doi:10.1016/0197-2456(86)90046-2

24 Adewuyi SA, Ajekigbe AT, Campbell OB, Mbibu NH, Oguntayo AO, Kolawole AO, et al. Pattern of oncologic emergencies seen in adult cancer patients attending the Radiotherapy and Oncology Centre, Ahmadu Bello University Teaching Hospital, Zaria - Nigeria. Niger Postgrad Med J. 2012;19:208-14. Medline:23385675

25 Bedwani R, Abdel-Fattah M, El-Shazly M, Bassili A, Zaki A, Seif HA, et al. Profile of familial breast cancer in Alexandria, Egypt. Anticancer Res. 2001;21:3011-4. Medline:11712803

26 Bodalal Z, Azzuz R, Bendardaf R. Cancers in Eastern Libya: first results from Benghazi Medical Center. World J Gastroenterol. 2014;20:6293-301. Medline:24876750 doi:10.3748/wjg.v20.i20.6293

27 Bouchbika Z, Haddad H, Benchakroun N, Eddakaoui H, Kotbi S, Megrini A, et al. Cancer incidence in Morocco: report from Casablanca registry 2005-2007. Pan Afr Med J. 2013;16:31. Medline:24570792 doi:10.11604/pamj.2013.16.31.2791 
28 Bouchlaka A, Ben Abdallah M, Ben Aissa R, Smida S, Ouechtati A, Boussen H, et al. Practice of large scale mammography in the Ariana area of Tunisia: prelude to a mass screening? Tunis Med. 2009;87:426-31. Medline:20063674

29 Calys-Tagoe BN, Yarney J, Kenu E, Amanhyia NA, Enchill E, Obeng I. Profile of cancer patients' seen at Korle Bu teaching hospital in Ghana (a cancer registry review). BMC Res Notes. 2014;7:577. Medline:25164384 doi:10.1186/17560500-7-577

30 Chbani L, Hafid I, Berraho M, Mesbahi O, Nejjari C, Amarti A. Epidemiological and pathological features of cancer in Fez Boulemane region, Morocco. East Mediterr Health J. 2013;19:263-70. Medline:23879078 doi:10.26719/2013.19.3.263

31 Chokunonga E, Borok MZ, Chirenje ZM, Nyakabau AM, Parkin DM. Trends in the incidence of cancer in the black population of Harare, Zimbabwe 1991-2010. Int J Cancer. 2013;133:721-9. Medline:23364833 doi:10.1002/ijc.28063

32 Dem A, Traore B, Dieng MM, Diop PS, Ouajdi T, Lalami MT, et al. Gynaecological and breast cancers at the Dakar Cancer Institute. Sante. 2008;18:25-9. Medline:18684687

33 Denewer A, Hussein O, Farouk O, Elnahas W, Khater A, El-Saed A. Cost-effectiveness of clinical breast assessment-based screening in rural Egypt. World J Surg. 2010;34:2204-10. Medline:20533039 doi:10.1007/s00268-010-0620-3

34 El Fakir S, Najdi A, Khazraji YC, Bennani M, Belakhel L, Abousselham L, et al. Breast Cancer Screening in Morocco: Performance Indicators During Two Years of an Organized Programme. Asian Pac J Cancer Prev. 2015;16:6285-8. Medline:26434830 doi:10.7314/APJCP.2015.16.15.6285

35 El Mistiri M, Salati M, Marcheselli L, Attia A, Habil S, Alhomri F, et al. Cancer incidence, mortality, and survival in Eastern Libya: updated report from the Benghazi Cancer Registry. Ann Epidemiol. 2015;25:564-8. Medline:25911981 doi:10.1016/j.annepidem.2015.03.012

36 El Mistiri M, Verdecchia A, Rashid I, El Sahli N, El Mangush M, Federico M. Cancer incidence in eastern Libya: the first report from the Benghazi Cancer Registry, 2003. Int J Cancer. 2007;120:392-7. Medline:17066425 doi:10.1002/ijc.22273

37 Enow Orock GE, Ndom P, Doh AS. Current cancer incidence and trends in Yaounde, Cameroon. Oncol Gastroenterol Hepatol Reports. 2012;1:58-63. doi:10.4103/2348-3113.133639

38 Garba SM, Zaki HM, Arfaoui A, Hami H, Soulaymani A, Nouhou H, et al. Epidemiology of cancers in Niger, 1992 to 2009. Bull Cancer. 2013;100:127-33. Medline:23420007

39 Hamdi Cherif M, Serraino D, Mahnane A, Laouamri S, Zaidi Z, Boukharouba H, et al. Time trends of cancer incidence in Setif, Algeria, 1986-2010: an observational study. BMC Cancer. 2014;14:637. Medline:25175348 doi:10.1186/14712407-14-637

40 Jedy-Agba E, Curado MP, Ogunbiyi O, Oga E, Fabowale T, Igbinoba F, et al. Cancer incidence in Nigeria: a report from population-based cancer registries. Cancer Epidemiol. 2012;36:e271-8. Medline:22621842 doi:10.1016/j. canep.2012.04.007

41 Korir A, Okerosi N, Ronoh V, Mutuma G, Parkin M. Incidence of cancer in Nairobi, Kenya (2004-2008). Int J Cancer. 2015;137:2053-9. Medline:26139540 doi:10.1002/ijc.29674

42 Laryea DO, Awuah B, Amoako YA, Osei-Bonsu E, Dogbe J, Larsen-Reindorf R, et al. Cancer incidence in Ghana, 2012: evidence from a population-based cancer registry. BMC Cancer. 2014;14:362. Medline:24884730 doi:10.1186/14712407-14-362

43 Lopes LV, Miguel F, Freitas H, Tavares A, Pangui S, Castro C, et al. Stage at presentation of breast cancer in Luanda, Angola - a retrospective study. BMC Health Serv Res. 2015;15:471. Medline:26471845 doi:10.1186/s12913-015-1092-9

44 Lorenzoni C, Vilajeliu A, Carrilho C, Ismail MR, Castillo P, Augusto O, et al. Trends in cancer incidence in Maputo, Mozambique, 1991-2008. PLoS One. 2015;10:e0130469. Medline:26110774 doi:10.1371/journal.pone.0130469

45 Maalej M, Hentati D, Messai T, Kochbati L, El May A, Mrad K, et al. Breast cancer in Tunisia in 2004: a comparative clinical and epidemiological study. Bull Cancer. 2008;95:E5-9. Medline:18304901

46 Missaoui N, Landolsi H, Jaidaine L, Ben Abdelkader A, Yaacoubi MT, Hmissa S. Breast cancer in central Tunisia: an earlier age at diagnosis and incidence increase over a 15-year period. Breast J. 2012;18:289-91. Medline:22487378 doi:10.1111/j.1524-4741.2012.01242.x

47 Mohammed AZ, Edino ST, Ochicha O, Gwarzo AK, Samaila AA. Cancer in Nigeria: a 10-year analysis of the Kano cancer registry. Niger J Med. 2008;17:280-4. Medline:18788253 doi:10.4314/njm.v17i3.37396

48 Msyamboza KP, Dzamalala C, Mdokwe C, Kamiza S, Lemerani M, Dzowela T, et al. Burden of cancer in Malawi; common types, incidence and trends: national population-based cancer registry. BMC Res Notes. 2012;5:149. Medline:22424105 doi:10.1186/1756-0500-5-149

49 Cancer Association of South Africa. South African Cancer Statistics. South Africa: CANSA; 2016 Available: http://www. cansa.org.za/south-african-cancer-statistics/. Accessed: 22 August 2016.

50 Nayama M, Nouhou H, Souna-Madougou K, Idi N, Garba M, Tahirou A, et al. Epidemiological and histological aspects of gynecologic and breast cancer in the pathology department of Niamey's Health Faculty, Niger. Mali Med. 2006;21:439. Medline: 19435008

51 Nggada HA, Yawe KD, Abdulazeez J, Khalil MA. Breast cancer burden in Maiduguri, North eastern Nigeria. Breast J. 2008;14:284-6. Medline:18476884 doi:10.1111/j.1524-4741.2008.00576.x

52 Nguefack CT, Biwole ME, Massom A, Kamgaing JT, Njamen TN, Ekane GH, et al. Epidemiology and surgical management of breast cancer in gynecological department of Douala General Hospital. Pan Afr Med J. 2012;13:35. Medline:23330026

53 Ntekim A, Nufu FT, Campbell OB. Breast cancer in young women in Ibadan, Nigeria. Afr Health Sci. 2009;9:242-6. Medline:21503175

54 Ohene-Yeboah M, Adjei E. Breast cancer in Kumasi, Ghana. Ghana Med J. 2012;46:8-13. Medline:22605883

55 Pace LE, Dusengimana JM, Hategekimana V, Habineza H, Bigirimana JB, Tapela N, et al. Benign and malignant breast disease at Rwanda's first public cancer referral center. Oncologist. 2016;21:571-5. Medline:27009935 doi:10.1634/theoncologist.2015-0388 
56 Parkin DM, Sitas F, Chirenje M, Stein L, Abratt R, Wabinga H, et al. Cancer in Indigenous Africans-burden, distribution, and trends. Lancet Oncol. 2008;9:683-92. Medline:18598933 doi:10.1016/S1470-2045(08)70175-X

57 Rahman GA, Olatoke SA, Agodirin SO, Adeniji KA. Socio-demographic and clinical profile of immuno-histochemically confirmed breast cancer in a resource limited country. Pan Afr Med J. 2014;17:182. Medline:25392728 doi:10.11604/ pamj.2014.17.182.2257

58 Saeed IE, Weng HY, Mohamed KH, Mohammed SI. Cancer incidence in Khartoum, Sudan: first results from the Cancer Registry, 2009-2010. Cancer Med. 2014;3:1075-84. Medline:24821265 doi:10.1002/cam4.254

59 Sighoko D, Bah E, Haukka J, McCormack VA, Aka EP, Bourgeois D, et al. Population-based breast (female) and cervix cancer rates in the Gambia: evidence of ethnicity-related variations. Int J Cancer. 2010;127:2248-56. Medline:20162609 doi:10.1002/ijc.25244

60 Somdyala NI, Bradshaw D, Gelderblom WC, Parkin DM. Cancer incidence in a rural population of South Africa, 19982002. Int J Cancer. 2010;127:2420-9. Medline:20162610 doi:10.1002/ijc.25246

61 Tonato Bagnan JA, Denakpo JL, Aguida B, Hounkpatin L, Lokossou A, De Souza J, et al. Epidemiology of the gynecological and mammary cancer to the HOMEL and in the CUGO Cotonou, Benin. Bull Cancer. 2013;100:141-6. Medline:23449498

62 Traoré B, Keita M, Diane S, Dankoro A, Kabba IS, Keita N. Clinicopathological study of breast diseases presenting to the surgical oncology unit of Donka University Hospital in Conakry, Guinea. West Afr J Med. 2012;31:227-31. Medline:23468023

63 Wabinga HR, Nambooze S, Amulen PM, Okello C, Mbus L, Parkin DM. Trends in the incidence of cancer in Kampala, Uganda 1991-2010. Int J Cancer. 2014;135:432-9. Medline:24615279 doi:10.1002/ijc.28661

64 Znati K, Bennis S, Abbass F, Akasbi Y, Chbani L, Elfatemi H, et al. Breast cancer in young patient in Morocco. Gynecol Obstet Fertil. 2014;42:149-54. Medline:22521987 doi:10.1016/j.gyobfe.2011.08.014

65 Adebamowo CA, Akarolo-Anthony S. Cancer in Africa: opportunities for collaborative research and training. Afr J Med Med Sci. 2009;38 Suppl 2:5-13. Medline:20229733

66 Akarolo-Anthony SN, Ogundiran TO, Adebamowo CA. Emerging breast cancer epidemic: evidence from Africa. Breast Cancer Res. 2010;12 Suppl 4:S8. Medline:21172092 doi:10.1186/bcr2737

67 Corbex M, Bouzbid S, Boffetta P. Features of breast cancer in developing countries, examples from North-Africa. Eur J Cancer. 2014;50:1808-18. Medline:24767469 doi:10.1016/j.ejca.2014.03.016

68 Huo D, Ikpatt F, Khramtsov A, Dangou JM, Nanda R, Dignam J, et al. Population differences in breast cancer: survey in indigenous African women reveals over-representation of triple-negative breast cancer. J Clin Oncol. 2009;27:4515-21. Medline:19704069 doi:10.1200/JCO.2008.19.6873

69 Baquet CR, Mishra SI, Commiskey P, Ellison GL, DeShields M. Breast cancer epidemiology in blacks and whites: disparities in incidence, mortality, survival rates and histology. J Natl Med Assoc. 2008;100:480-8. Medline:18507200 doi:10.1016/S0027-9684(15)31294-3

70 Dickens C, Pfeiffer RM, Anderson WF, Duarte R, Kellett P, Schuz J, et al. Investigation of breast cancer sub-populations in black and white women in South Africa. Breast Cancer Res Treat. 2016;160:531-7. Medline:27757717 doi:10.1007/ s10549-016-4019-1

71 Fregene A, Newman LA. Breast cancer in sub-Saharan Africa: how does it relate to breast cancer in African-American women? Cancer. 2005;103:1540-50. Medline:15768434 doi:10.1002/cncr.20978

72 Hortobagyi GN, de la Garza Salazar J, Pritchard K, Amadori D, Haidinger R, Hudis CA, et al. The global breast cancer burden: variations in epidemiology and survival. Clin Breast Cancer. 2005;6:391-401. Medline:16381622 doi:10.3816/ CBC.2005.n.043

73 Balekouzou A, Yin P, Afewerky HK, Bekolo C, Pamatika CM, Nambei SW, et al. Behavioral risk factors of breast cancer in Bangui of Central African Republic: A retrospective case-control study. PLoS One. 2017;12:e0171154. Medline:28178283 doi:10.1371/journal.pone.0171154

74 Adeloye D, David RA, Aderemi AV, Iseolorunkanmi A, Oyedokun A, Iweala EEJ, et al. An Estimate of the Incidence of Prostate Cancer in Africa: A Systematic Review and Meta-Analysis. PLoS One. 2016;11:e0153496. Medline:27073921 doi:10.1371/journal.pone.0153496

75 Balekouzou A, Yin P, Pamatika CM, Bishwajit G, Nambei SW, Djeintote M, et al. Epidemiology of breast cancer: retrospective study in the Central African Republic. BMC Public Health. 2016;16:1230. Medline:27923361 doi:10.1186/ s12889-016-3863-6

76 Kantelhardt EJ, Frie KG. How advanced is breast cancer in Africa? Lancet Glob Health. 2016;4:e875-6. Medline:27855857 doi:10.1016/S2214-109X(16)30283-2

77 Jones CEL, Maben J, Jack RH, Davies EA, Forbes LJL, Lucas G, et al. A systematic review of barriers to early presentation and diagnosis with breast cancer among black women. BMJ Open. 2014;4:e004076. Medline:24523424 doi:10.1136/ bmjopen-2013-004076

78 Abdulrahman GO Jr, Rahman GA. Epidemiology of breast cancer in Europe and Africa. J Cancer Epidemiol. 2012;2012:915610. Medline:22693503 doi:10.1155/2012/915610

79 Jedy-Agba EE, Curado MP, Oga E, Samaila MO, Ezeome ER, Obiorah C, et al. The role of hospital-based cancer registries in low and middle income countries-The Nigerian Case Study. Cancer Epidemiol. 2012;36:430-5. Medline:22704971 doi:10.1016/j.canep.2012.05.010

80 Singh E, Sengayi M, Urban M, Babb C, Kellett P, Ruff P. The South African National Cancer Registry: an update. Lancet Oncol. 2014;15:e363. Medline:25079099 doi:10.1016/S1470-2045(14)70310-9 